This item was submitted to Loughborough's Research Repository by the author.

Items in Figshare are protected by copyright, with all rights reserved, unless otherwise indicated.

\title{
Frontogenesis in gravity-driven flows with non-uniform density gradients
}

PLEASE CITE THE PUBLISHED VERSION

PUBLISHER

(c) Cambridge University Press

VERSION

VoR (Version of Record)

LICENCE

CC BY-NC-ND 4.0

REPOSITORY RECORD

Kay, Anthony. 2019. "Frontogenesis in Gravity-driven Flows with Non-uniform Density Gradients". figshare. https://hdl.handle.net/2134/4260. 
This item was submitted to Loughborough's Institutional Repository (https://dspace.lboro.ac.uk/) by the author and is made available under the following Creative Commons Licence conditions.

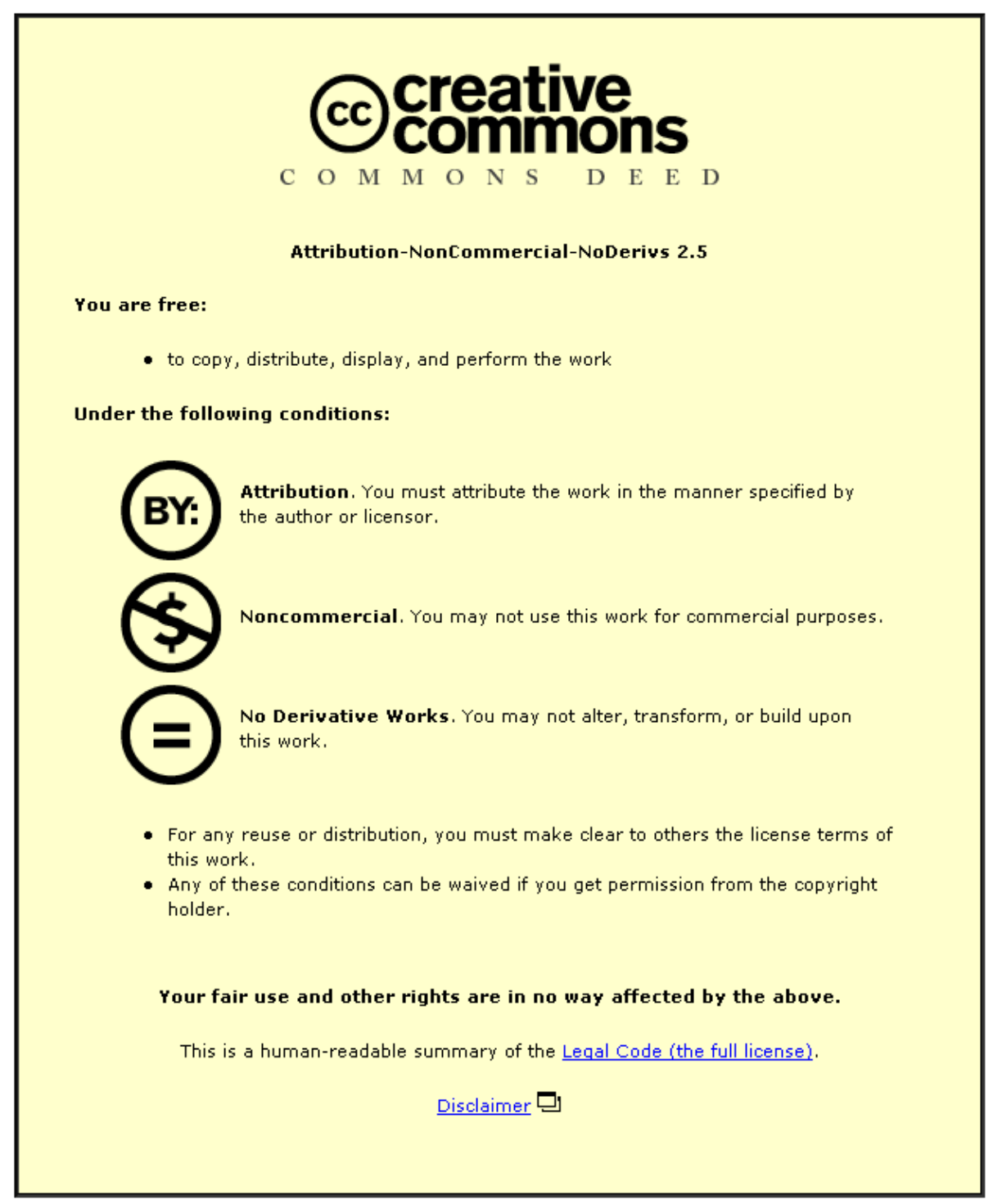

For the full text of this licence, please go to: http://creativecommons.org/licenses/by-nc-nd/2.5/ 


\title{
Frontogenesis in gravity-driven flows with non-uniform density gradients
}

\author{
By ANTHONY KAY \\ Department of Mathematical Sciences, Loughborough University of Technology, \\ Loughborough, Leicestershire, LE11 3TU, UK
}

(Received 21 September 1990 and in revised form 13 August 1991)

A horizontal density gradient may be steepened to form a front if the horizontal flow which it drives is convergent. This convergence may be caused by an initial nonlinearity in the density gradient (as described by Simpson \& Linden 1989). A quadratic density profile is analysed to illustrate the mechanism, and it is shown how the flow and the density profile interact to intensify and concentrate the front near a horizontal boundary. Linear and curved density profiles in a container of finite length are also studied: the most favourable location for frontogenesis is found to be where the flow emerges into a region of significant curvature after passing through a maximum of the density gradient.

\section{Introduction}

Gravity currents are flows which are driven by hydrostatic pressure differences resulting from horizontal density variations in the fluid. They are frequently observed to have a frontal structure (Simpson 1982); this is not surprising in cases where the initial density is a step function of the horizontal coordinate, as in lockexchange experiments or dense gas releases. However, the formation of a front is often observed in situations where the initial horizontal density variation is continuous (Linden \& Simpson 1986, hereafter referred to as LS) : examples include salinity fronts in estuaries and sea-breeze fronts.

The mechanism for this frontogenesis was elucidated by Simpson \& Linden (1989, hereafter referred to as SL). Fundamental to SL's theory is their equation (3.10) for the evolution of the horizontal density gradient:

$$
\frac{\partial}{\partial t}\left(\frac{\partial \rho}{\partial x}\right)=-\frac{\partial}{\partial x}\left(u \frac{\partial \rho}{\partial x}\right)
$$

(derived by differentiating the incompressible density equation

$$
\mathrm{D} \rho / \mathrm{D} t=0
$$

with respect to $x$ and ignoring vertical motion). The mechanisms described by (1.1) become clearer if it is expanded:

$$
\frac{\partial}{\partial t}\left(\frac{\partial \rho}{\partial x}\right)=-u \frac{\partial^{2} \rho}{\partial x^{2}}-\frac{\partial u}{\partial x} \frac{\partial \rho}{\partial x} .
$$

The term $-u \partial^{2} \rho / \partial x^{2}$ represents the advection of fluid with a different density gradient past a stationary observer, and does not therefore imply any change to the density gradient in the fluid. (Throughout this paper, the term 'density gradient' will refer to horizontal density gradients; a vertical density gradient will be denoted by the term 'stratification'.) The term $-(\partial u / \partial x)(\partial \rho / \partial x)$ represents the steepening or 


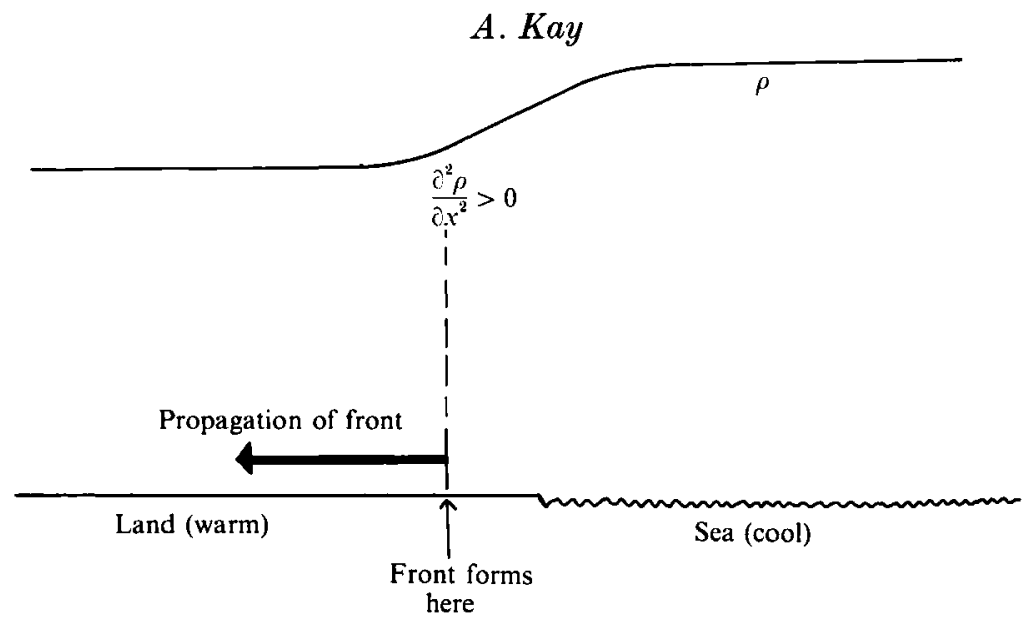

FIGURE 1. Horizontal density variation responsible for the formation and propagation of a seabreeze front.

flattening of the density gradient as neighbouring isopycnals moving with different horizontal velocities are bunched together or pushed apart. In particular, where the horizontal flow is convergent $(\partial u / \partial x<0)$ the gradient will steepen, possibly to the extent of forming a front. Note that the terms 'convergent' and 'divergent' will be used in this paper to refer only to the horizontal component of flow, i.e. to denote non-zero $\partial u / d x$; we take $\boldsymbol{\nabla} \cdot \boldsymbol{u}=\mathbf{0}$ throughout. The association between horizontal convergence and frontogenesis is well-known in meteorology (Hoskins 1982). Convergence of the horizontal flow occurs where the strength of the gravity current varies with the horizontal coordinate. Such a non-uniformity of the flow may result from a non-uniformity of the initial density gradient which is responsible for the flow : SL deduced that a front will form where the flow is from a region of strong density gradient to a region of weaker gradient, and will then propagate with the flow, as in a sea breeze (figure 1).

Gravity currents may be described in terms of baroclinic generation of vorticity,

$$
\frac{\mathrm{D} \omega}{\mathrm{D} t}=\frac{\mathrm{g}}{\bar{\rho}} \frac{\partial \rho}{\partial x}
$$

Here we have assumed two-dimensional, inviscid, Boussinesq flow; $\bar{\rho}$ is a reference density. In all geophysical flows and in the experiments carried out by LS and SL, horizontal lengthscales are much greater than the vertical extent of the flow; hence

$$
\omega \approx \frac{\partial u}{\partial z}
$$

Differentiating (1.3) with respect to $x$ and using (1.4),

$$
\frac{\mathrm{D}}{\mathrm{D} t}\left(\frac{\partial}{\partial z}\left(\frac{\partial u}{\partial x}\right)\right) \approx \frac{g}{\bar{\rho}} \frac{\partial^{2} \rho}{\partial x^{2}}
$$

The expected location for frontogenesis is where the convergence (negative $\partial u / \partial x$ ) is greatest; the vertical derivative on the left-hand side of (1.5) suggests that this will be at the lower boundary if the curvature of the density profile $\left(\partial^{2} \rho / \partial x^{2}\right)$ is positive, and at the upper boundary if $\partial^{2} \rho / \partial x^{2}$ is negative. This prediction is consistent with SL's ideas on the location of frontogenesis; it is confirmed by their experimental results with a piecewise-linear initial density profile (i.e. with $\partial^{2} \rho / \partial x^{2}$ a delta-function of $x$ ) and by observations of frontogenesis in sea breezes (see figure 1 ). 
The aim of the present work is to investigate further the process by which curvature of the density profile leads to steepening of the density gradient. We shall consider in $\S 3$ the case of a quadratic profile (i.e. with $\partial^{2} \rho / \partial x^{2}$ uniform), which may be motivated physically by the existence of the density maximum at $4{ }^{\circ} \mathrm{C}$ in fresh water; thus a linear temperature profile around $4{ }^{\circ} \mathrm{C}$ would give the required quadratic density profile. SL have noted that vertical flow is associated with curvature in the density profile (since continuity necessitates that a convergent horizontal flow must be accompanied by some vertical motion); we shall see that uniform $\partial^{2} \rho / \partial x^{2}$ induces a vertical flow which is also uniform (with respect to the horizontal coordinate). Hence (1.4) becomes an exact equation, and the resulting algebraic simplifications will facilitate a deeper analysis of the frontogenesis process than SL were able to undertake. The relevance of the results to the dynamics of the thermal bar (the front which is observed around the $4^{\circ} \mathrm{C}$ isotherm in lakes) will be considered.

The analysis for a uniformly curved density profile will assume the flow to be of infinite horizontal extent; similarly, SL ignored the effect of endwalls when analysing linear and piecewise-linear profiles. However, LS ascribed the frontogenesis in their experiments to the curvature which must exist somewhere in the density profile in order to satisfy the diffusive zero-flux boundary condition (on salinity, to which density is linearly related) at the endwalls. We shall therefore investigate the formation of fronts in a fluid contained in a tank of finite length with initial density profiles similar to those observed by LS (see figure 13 below and LS's figure 9). The case where there is an initial stable stratification as well as a horizontal density gradient will also be analysed: the initial conditions in SL's experiments included a stratification $\pi$ times stronger than the horizontal density gradient (whereas the fluid in LS's tank was vertically well-mixed when frontogenesis was initiated).

\section{Mathematical formulation}

To study the dynamics of a gravity current, we need to simultaneously consider the evolution of the density field and the flow. Ignoring diffusion, the density equation in two-dimensional flow is

$$
\frac{\partial \rho}{\partial t}=-u \frac{\partial \rho}{\partial x}-w \frac{\partial \rho}{\partial z}
$$

the vorticity equation (1.3) may be expanded as

$$
\frac{\partial \omega}{\partial t}=-u \frac{\partial \omega}{\partial x}-w \frac{\partial \omega}{\partial z}+\frac{g}{\bar{\rho}} \frac{\partial \rho}{\partial x},
$$

and the flow velocity components are

$$
u=-\frac{\partial \psi}{\partial z}, \quad w=\frac{\partial \psi}{\partial x}
$$

The stream function is obtained by solving

$$
\nabla^{2} \psi=-\omega
$$

with $\psi=\mathbf{0}$ on rigid boundaries. The density of a fluid particle has been assumed to be a function only of temperature or salinity, but the equation of state is immaterial until diffusion is taken into consideration. 
When heat (or salt) is allowed to diffuse, we must replace (2.1) with an advection-diffusion equation,

$$
\frac{\partial T}{\partial t}=-u \frac{\partial T}{\partial x}-w \frac{\partial T}{\partial z}+\kappa\left(\frac{\partial^{2} T}{\partial x^{2}}+\frac{\partial^{2} T}{\partial z^{2}}\right)
$$

where $\kappa$ is an isotropic diffusivity and $T$ can be either temperature or salinity. If the equation of state is linear, the density equation takes the same form as (2.5), but in other cases it is more convenient to stay with $T$ as the 'fluid state' variable. For consistency, diffusion of vorticity (i.e. viscous effects) must also be accounted for, by the addition of a term

$$
\nu\left(\frac{\partial^{2} \omega}{\partial x^{2}}+\frac{\partial^{2} \omega}{\partial z^{2}}\right)
$$

(where $v$ is the kinematic viscosity) to the right-hand side of the vorticity equation (2.2). The last term in (2.2) can be written as

$$
\frac{g}{\rho} \frac{\partial \rho}{\partial T} \frac{\partial T}{\partial x}
$$

(where $\partial \rho / \partial T$ is known from the equation of state) when $T$ is used as the fluid state variable.

Since we shall be considering flows accelerating rapidly from rest, the dominant dynamical balance will be between inertia and buoyancy forces; thus the analysis in $\$ 3$ (for a uniformly curved density profile) will use (2.1)-(2.4), and the effects of diffusion will only be mentioned briefly. When considering gravity currents in a tank of finite length (in $\S 4$ ) we will suppose that diffusion plays a role in setting up an initial density profile, but the subsequent evolution of the flow will again be modelled without diffusive effects.

We use SL's method of solution, in which the field variables are expanded as power series in time $t$,

$$
\left.\begin{array}{l}
\omega=\omega_{0}+\omega_{1} t+\omega_{2} t^{2}+\ldots, \\
\rho=\rho_{0}+\rho_{1} t+\rho_{2} t^{2}+\ldots, \text { etc. }
\end{array}\right\}
$$

These expansions are substituted into the evolution equations, and it is straightforward to extract the density and vorticity perturbations at each order in $t$ (although from (2.1), (2.2) and the initial condition of fluid at rest, it can be shown that only even-order perturbations to the density field and odd-order perturbations to the flow will be non-zero); the only difficulties that may arise are in the solution of (2.4) at each order. This method can yield useful information on how and where frontogenesis starts in gravity currents; to trace the full development of a front requires a numerical solution of the evolution equations, which has so far been done only for the case of a uniformly curved density profile. Furthermore, the method of expansion in powers of $t$ can only describe developments in situ; it cannot trace the propagation of a front through the fluid; nor would it admit the satisfaction of the boundary conditions on $T$ or $\rho$ if diffusive effects were included.

\section{Quadratic density profile}

3.1. Solution by expansion in powers of $t$

We consider the development of the flow generated by an initial density profile

$$
\rho_{0}=\bar{\rho}-\gamma x^{2},
$$




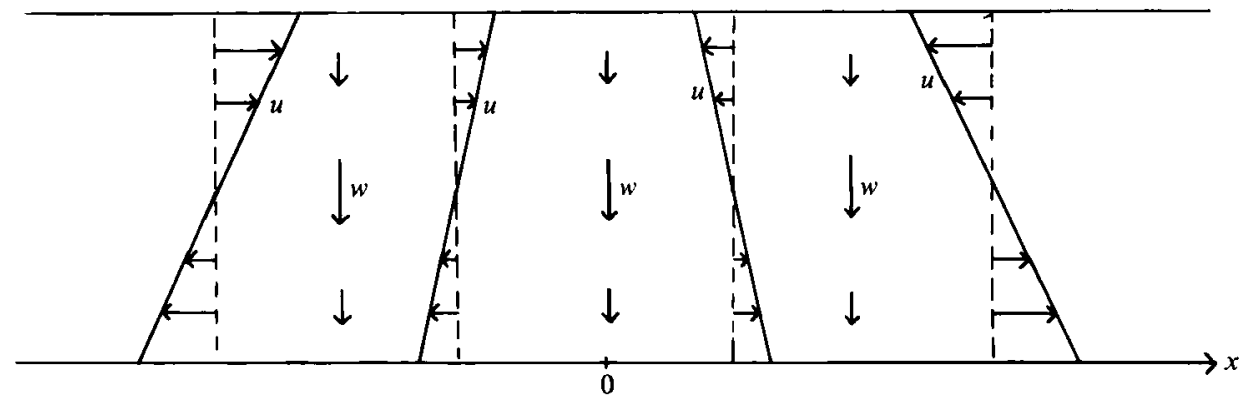

FIGURE 2. $O(t)$ flow driven by a quadratic density profile, with profiles for horizontal velocity shown schematically.

where $\gamma$ depends on the equation of state and the initial temperature profile. For an initially stationary fluid, $(2.2)$ yields at $O(1)$ in $t$

$$
\omega_{1}=\frac{g}{\bar{\rho}} \frac{\partial \rho_{0}}{\partial x}=-\frac{2 g \gamma x}{\bar{\rho}} .
$$

We now seek velocity components for the flow in a region of infinite horizontal extent and with rigid boundaries at $z= \pm \frac{1}{2} h$, such that the vorticity is given by (3.2) and the vertical velocity is independent of $x$ (as suggested in $\S 1$ above). Thus

$$
\frac{\partial u_{1}}{\partial z}=-\frac{2 g \gamma x}{\bar{\rho}} \text { with } \int_{-\frac{1}{2} h}^{\frac{1}{2} h} u_{1} \mathrm{~d} z=\text { constant }
$$

and symmetry (about $x=0$ ) indicates that the constant is zero; hence

$$
u_{1}=-\frac{2 g \gamma x z}{\bar{\rho}}
$$

Continuity then yields

$$
w_{1}=\frac{g \gamma}{\bar{\rho}}\left(z^{2}-\frac{h^{2}}{4}\right)
$$

(Note that the velocity components are $u_{1} t$ and $w_{1} t ; u_{1}$ and $w_{1}$ are components of the initial acceleration.) The horizontal flow is converging towards the density maximum in the upper half of the cavity, and diverging away from it in the lower half; there is a downward flow everywhere to compensate for this, as shown schematically in figure 2. The inviscid flow would be unaffected by a wall at $x=0$; because of the symmetry about this plane, streamlines for this flow have been plotted only for $x>0$ : they are the dashed lines in figure 3. Note that 'convergence' in the sense of negative $\partial u / \partial x$ shows up as streamlines which diverge along the flow direction.

The pattern of convergence and divergence leads us to expect the density gradient to steepen in $z>0$ and to flatten in $z<0$. Expanding the density equation (2.1) in powers of $t$, we obtain

$$
\rho_{2}=-\frac{1}{2} u_{1} \frac{\partial \rho_{0}}{\partial x}=-\frac{2 g \gamma^{2} x^{2} z}{\bar{\rho}} .
$$

(If there was an initial uniform stratification $\partial \rho_{0} / \partial z=-\beta$ as in SL's calculations, this would have no effect on the dynamics; for with $w$ independent of $x$, the extra term $-w \partial \rho_{0} / \partial z$ in (3.5) would be independent of $x$ and would have no effect on the flow 


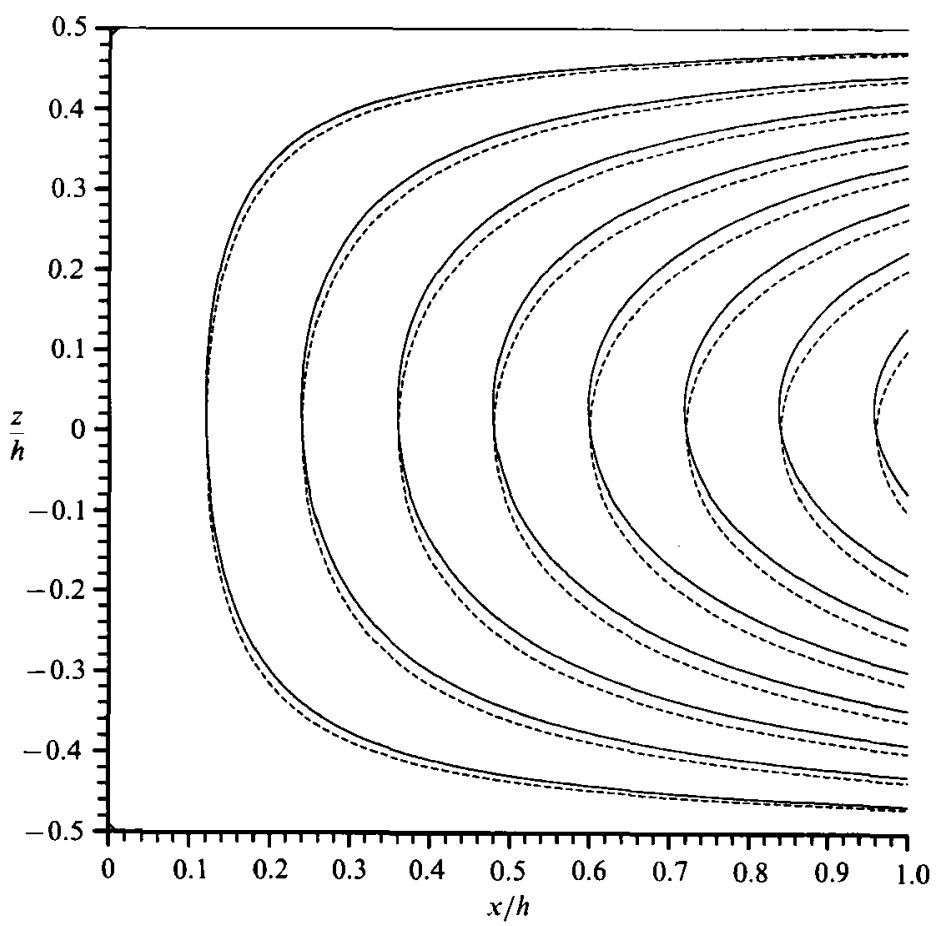

Figure 3. Streamlines at time $t=0.7(\bar{\rho} / g \gamma h)^{\frac{1}{2}}$ in the region $0<x<h$, for flow driven by a quadratic density profile: $\cdots . . . \cdot, O(t)$ flow only;,$- O(t)$ flow plus $O\left(t^{3}\right)$ perturbation.

which is driven by density gradients.) Equation (3.5) shows that, as in any gravity current, the flow is becoming stably stratified $\left(\partial \rho_{2} / \partial z<0\right.$ everywhere $)$; also, the perturbation to the horizontal density gradient is as expected, since

$$
\frac{\partial \rho}{\partial x}=\frac{\partial \rho_{0}}{\partial x}+\frac{\partial \rho_{2}}{\partial x} t^{2}+\ldots=-2 \gamma x\left(1+\frac{g \gamma z}{\bar{\rho}} t^{2}+\ldots\right),
$$

i.e. an increase in magnitude with $t$ for $z>0$, and a decrease for $z<0$. This is illustrated in figure 4 , in which isopycnals are plotted at times $t=0$ and $t=0.7 L_{\rho} /(g h)^{\frac{1}{2}}$, where $L_{\rho} \equiv(\bar{\rho} / \gamma)^{\frac{1}{2}}$ is the lengthscale for horizontal density variations.

According to (3.6) the timescale for frontogenesis is

$$
t_{\rho}=\left(\frac{\bar{\rho}}{g \gamma h}\right)^{\frac{1}{2}}=\frac{L_{\rho}}{(g h)^{\frac{1}{2}}},
$$

which differs by a factor of order $\left(L_{\rho} / h\right)^{\frac{1}{2}}$ from SL's estimate. The expansion in powers of $t$ can only be expected to give accurate results at times $t \leqslant t_{\rho}$. Furthermore, in any practical application, the horizontal extent of the region in which the density profile is quadratic will be finite; we could expect its length $L_{q}$ to be in the range $h \ll L_{q} \ll L_{\rho}$. Thus end effects will become important on a timescale

$$
t_{q} \sim\left(L_{q} / u_{1}\right)^{\frac{1}{2}}
$$

(since $u_{1}$ is the acceleration at $t=0$ ), so from (3.3) with $x \sim L_{q}$,

$$
t_{q} \sim\left(\frac{\bar{\rho}}{g \gamma h}\right)^{\frac{1}{2}}=t_{\rho}
$$




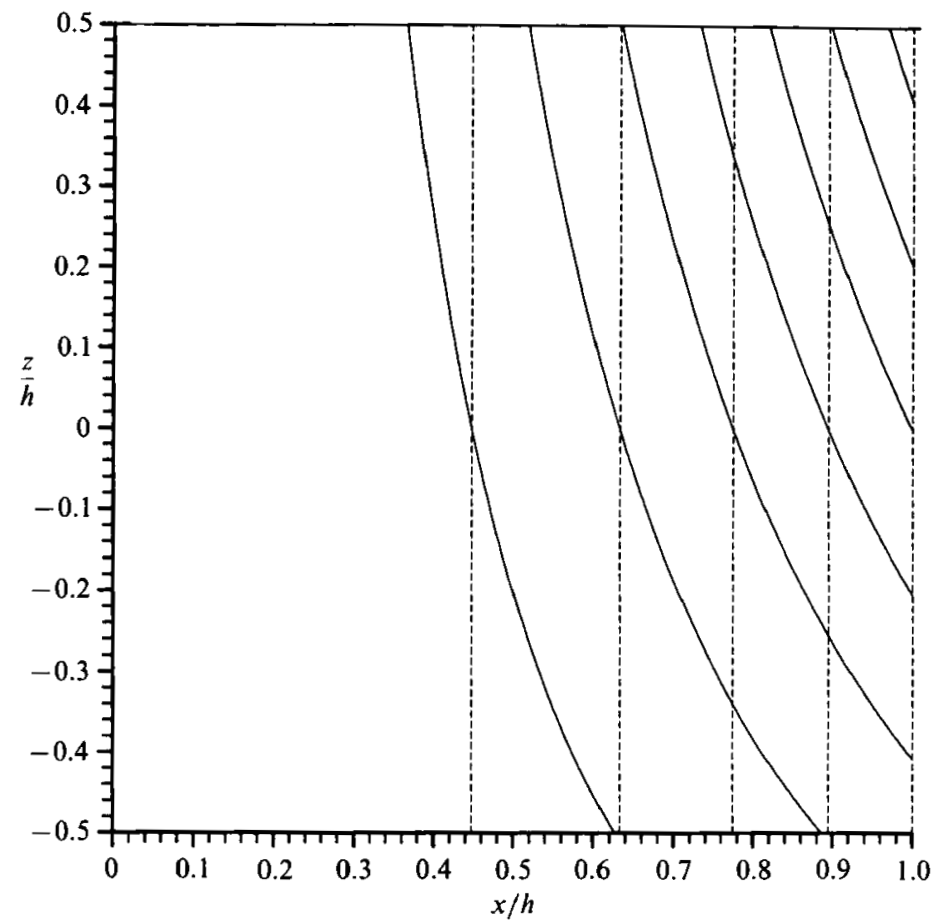

Fraure 4. Isopyenals in the region $0<x<h$ at $t=0$ (dashed lines) and $t=0.7(\bar{\rho} / g \gamma h)^{\frac{1}{2}}$ (solid lines) : density calculated as $\rho=\rho_{0}+\rho_{2} t^{2}$, using (3.1) and (3.5).

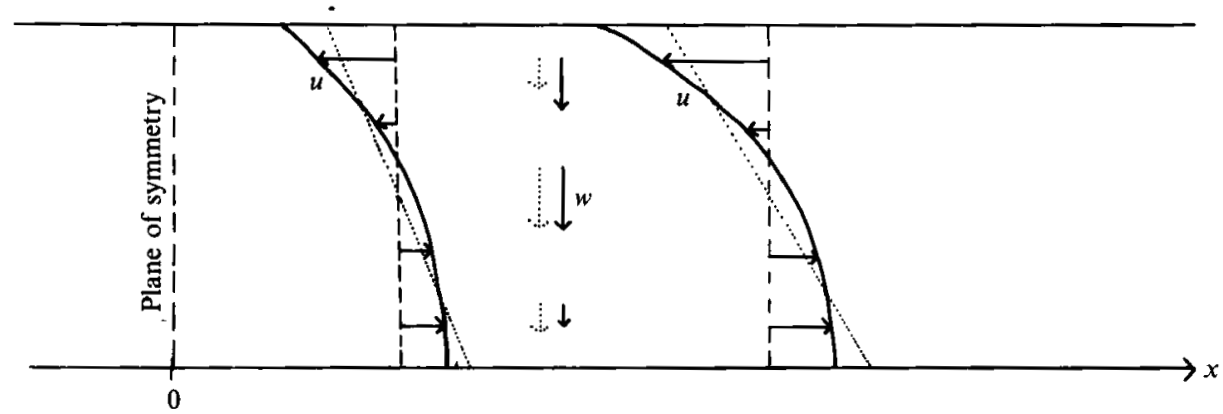

Figure 5. Effect of $O\left(t^{3}\right)$ flow perturbation on horizontal velocity profiles and vertical flow in $x>0$. Dotted lines represent flow at $O(t)$, solid lines include $O\left(t^{3}\right)$ flow.

The $O\left(t^{2}\right)$ perturbation to the density profile will in turn perturb the flow. The vorticity equation at $O\left(t^{2}\right)$ gives

$$
\omega_{3}=\frac{1}{3}\left(-u_{1} \frac{\partial \omega_{1}}{\partial x}-w_{1} \frac{\partial \omega_{1}}{\partial z}+\frac{g}{\bar{\rho}} \frac{\partial \rho_{2}}{\partial x}\right)=-\frac{8}{3}\left(\frac{g \gamma}{\bar{\rho}}\right)^{2} x z
$$

and we can find components of the velocity perturbation as before:

$$
\begin{aligned}
& u_{3}=(g \gamma / \bar{\rho})^{2} x\left(\frac{1}{9} h^{2}-\frac{4}{3} z^{2}\right), \\
& w_{3}=\frac{1}{9}(g \gamma / \bar{\rho})^{2} z\left(4 z^{2}-h^{2}\right) .
\end{aligned}
$$

The $O\left(t^{3}\right)$ changes to the horizontal velocity profile are illustrated schematically in figure 5 , and more precisely by plotting streamlines of $\psi_{1} t+\psi_{3} t^{3}$ at $t=0.7 L_{\rho} /(g h)^{\frac{1}{2}}$ as solid lines in figure 3 (for comparison with the $O(t)$ flow). From these diagrams and 


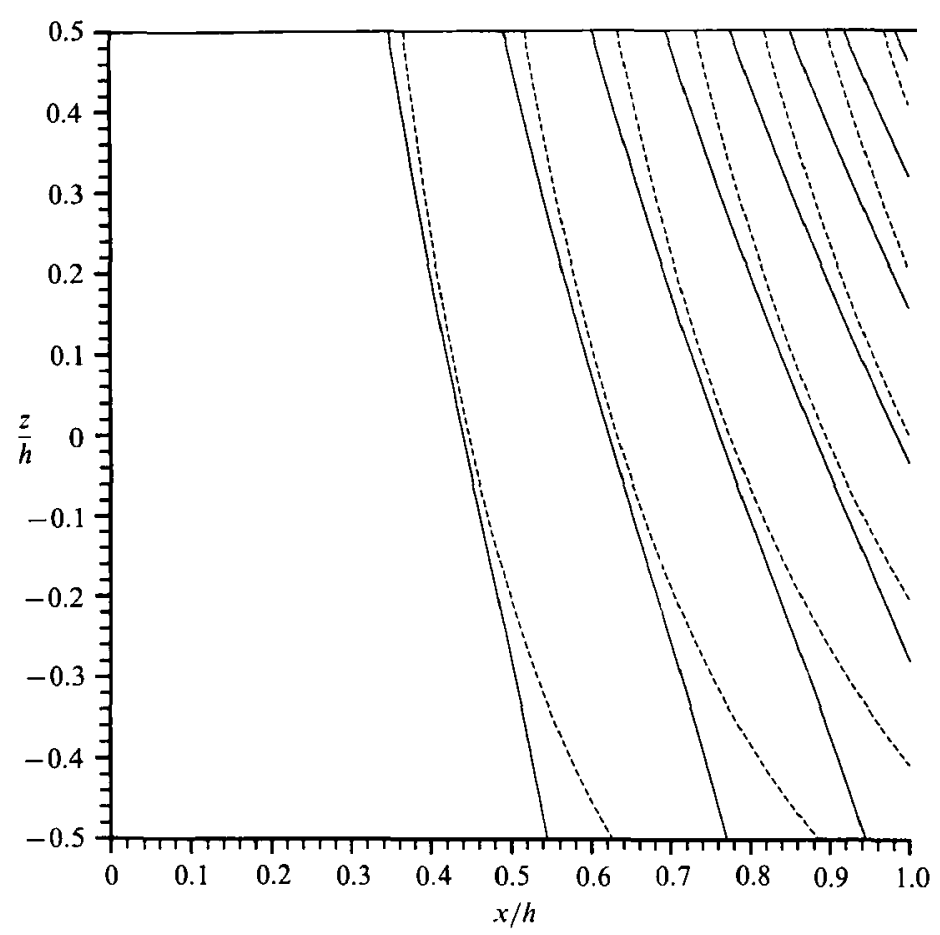

FIGURE 6. Effect of $O\left(t^{4}\right)$ density perturbation. Isopycnals at $t=0.7(\bar{\rho} / g \gamma h)^{\frac{1}{2}}$ : density calculated as $\rho=\rho_{0}+\rho_{2} t^{2}+\rho_{4} t^{4}$ (solid lines), and without $O\left(t^{4}\right)$ term (dashed lines).

(3.9), it can be seen that the shear $|\partial u / \partial z|$ and the convergence $(-\partial u / \partial x)$ of the horizontal flow are both increased near the upper boundary; these are expected features of the process of frontogenesis. The symmetry about the horizontal midplane has been broken; the positive feedback mechanism by which the convergence and shear of the $O(t)$ flow alters the density profile so as to intensify convergence and shear at $O\left(t^{3}\right)$ also concentrates these flow properties in a thinner layer near one boundary. This can again be seen if we calculate the $O\left(t^{4}\right)$ density perturbation,

$$
\rho_{4}=-\left(g^{2} \gamma^{2} x^{2} / 36 \bar{\rho}^{2}\right)\left(78 z^{2}+5 h^{2}\right),
$$

which indicates that both the horizontal density gradient and the stable stratification undergo their greatest increase near the upper boundary; this is illustrated in figure 6, which shows the effect of the $O\left(t^{4}\right)$ term (3.11) on the isopycnals at $t=0.7 L_{\rho} /(g h)^{\frac{1}{2}}$.

\subsection{Numerical solution of the evolution equations $\dagger$}

From (3.5) and (3.11) it is apparent that an initially quadratic density profile remains quadratic at all times; the density gradient steepens near the upper boundary, but no horizontal station is preferred above any other as a location for a front. (In practice, one might expect the exact location for frontogenesis to depend on end effects or any slight departures from the initial quadratic profile.) Similarly, the velocity components and the vorticity appear to retain the same form of $x$ -

† Calculations similar to those described in this section, and with similar results, have been done independently by Jacqmin (1991). He has also obtained interesting results from numerical integration of equations equivalent to (3.20) and (3.23). 
dependence at all times (compare (3.2)-(3.4) with (3.8)-(3.10)). We therefore seek to extract this constant $x$-dependence, defining a new set of field variables $\left(\rho^{*}, \omega^{*}, u^{*}, w^{*}\right)$ which are functions only of $z$ and $t$ :

$$
\begin{gathered}
\rho-\bar{\rho}=-\frac{1}{2} x^{2} \rho^{*}(z, t), \\
\omega=-x \omega^{*}(z, t), \\
u=-x u^{*}(z, t), \\
w=w^{*}(z, t) .
\end{gathered}
$$

Dimensionless variables may be defined using the timescale (3.7) and the first-order flow (3.2)-(3.4) to suggest scalings:

$$
\zeta=\frac{z}{h}, \quad \tau=t\left(\frac{g \gamma h}{\bar{\rho}}\right)^{\frac{1}{2}}, \quad R=\frac{\rho^{*}}{\gamma}, \quad \Omega=\omega^{*}\left(\frac{h \bar{\rho}}{g \gamma}\right)^{\frac{1}{2}}, \quad U=u^{*}\left(\frac{\bar{\rho}}{g \gamma h}\right)^{\frac{1}{2}}, \quad W=w^{*}\left(\frac{\bar{\rho}}{g \gamma h^{3}}\right)^{\frac{1}{2}} .
$$

In terms of these variables, the continuity equation and the definition of vorticity become

$$
\Omega=\frac{\partial U}{\partial \zeta}=\frac{\partial^{2} W}{\partial \zeta^{2}}
$$

The density and vorticity evolution equations (2.1) and (2.2) take the forms

$$
\begin{gathered}
\frac{\partial R}{\partial \tau}+W \frac{\partial R}{\partial \zeta}=2 U R \\
\frac{\partial \Omega}{\partial \tau}+W \frac{\partial \Omega}{\partial \zeta}=U \Omega+R
\end{gathered}
$$

thus they constitute a first-order quasi-linear system with a solution in terms of characteristics :

$$
\mathrm{d} \tau=\frac{\mathrm{d} \zeta}{W}=\frac{\mathrm{d} R}{2 U R}=\frac{\mathrm{d} \Omega}{U \Omega+R} .
$$

The initial conditions for this solution are

$$
\Omega=0 \text { and } R=2 \text { at } \tau=0 .
$$

Numerical integration along characteristics has been done according to a method described by Smith (1985); at each iteration at each time-step the value of $\Omega$ was interpolated between the characteristics with a Chebyshev polynomial so that integrations could be performed to evaluate $U$ and $W$ (as required by (3.16)). 21 characteristics were used, starting at $\zeta=\zeta_{0}^{(i)}(i=1, \ldots, 21)$ at $\tau=0$, with

$$
-0.5=\zeta_{0}^{(1)}<\ldots<\zeta_{0}^{(21)}=0.5 ;
$$

the spacing of the $\zeta_{0}^{(i)}$ was closest near the upper boundary where frontogenesis is expected to occur. These characteristics are plotted in figure 7, from which it is clear that the system approaches some kind of singularity at $\tau \approx 1.61$.

The quantities $U, W$ and $R$ (proportional to the horizontal flow convergence, the vertical velocity, and the density profile curvature, respectively) are plotted in figures 8,9 and 10 against the vertical coordinate $\zeta$ at dimensionless times $\tau=0.5$, 1.0, 1.2 and 1.3. (Similar curves, not shown here, have also been plotted using results 


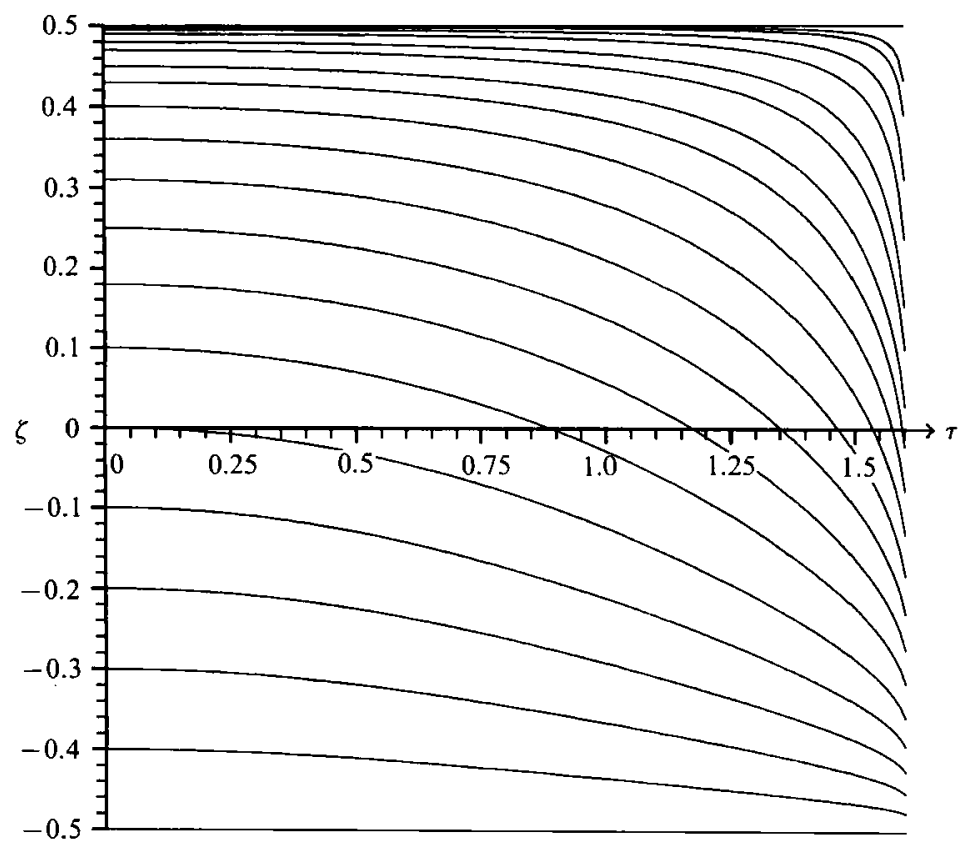

FiguRE 7. Characteristics $(\mathrm{d} \zeta=W \mathrm{~d} \tau)$ for equations (3.17) and (3.18).

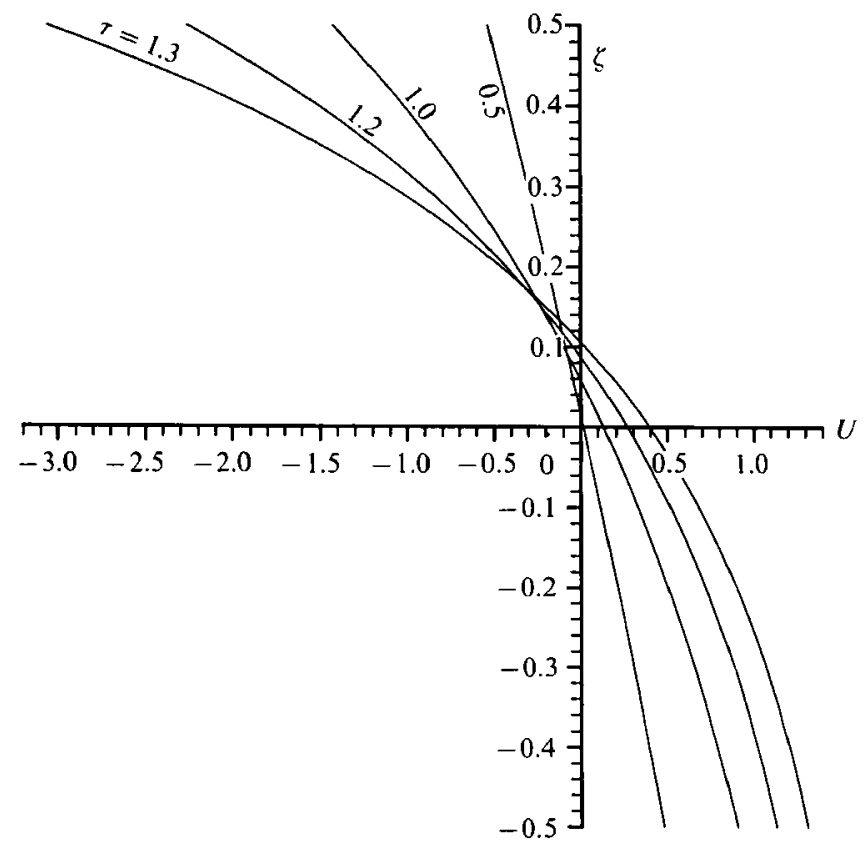

Figure 8. Vertical profiles of $U$ at dimensionless times $\tau=0.5,1.0,1.2,1.3$.

obtained from the expansion in powers of $t$ up to $O\left(t^{4}\right)$ : the two sets of curves are barely distinguishable at $\tau=0.5$, but there are significant differences at $\tau=1.0$.) Figure 8 shows the horizontal flow to be sheared almost uniformly at $\tau=0.5$ (cf. figure 2, showing the $O(t)$ horizontal flow), whereas at later times the shear becomes intensified increasingly rapidly near the upper boundary (cf. figure 5, the horizontal 


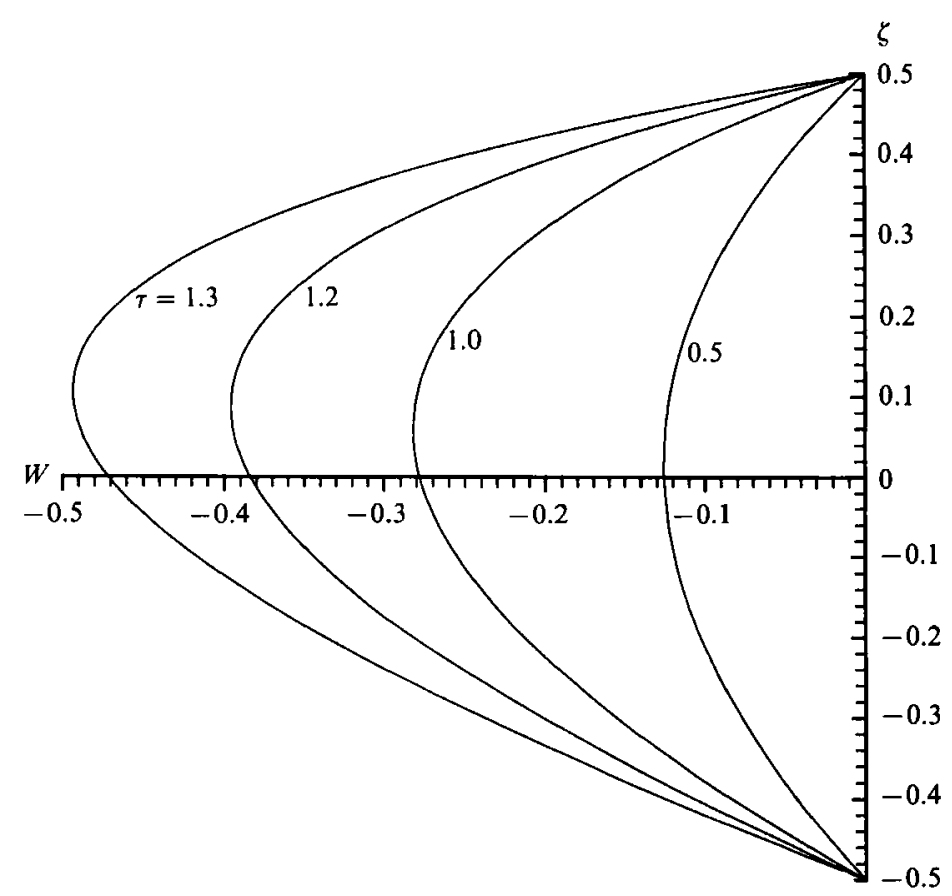

Figure 9. Vertical profiles of $W$ at dimensionless times $\tau=0.5,1.0,1.2,1.3$.

flow to $\left.O\left(t^{3}\right)\right)$. The intensification of the vertical flow is shown in figure 9 , in which the maximum of $W$ (which corresponds to the zero of $U$ ) is seen to move upwards with time; at $\tau=1.60$ (as close to the singularity as it has been possible to calculate), the position of this maximum reaches $\zeta=0.25$. The approach to a singularity in finite time is seen most dramatically in figure 10 , which shows how the steepening of density gradients near the upper boundary rapidly accelerates after $\tau=1.0$, while the gradient slackens in the lower part of the flow. Nearer the time of singularity, the value of $R$ at $\zeta=0.5$ increases to 1000 at $\tau=1.58,2300$ at $\tau=1.59$ and 8600 at $\tau=1.60$.

The finite-time blow-up $(R \rightarrow \infty$ at $\zeta=0.5$ at $\tau \approx 1.61)$ indicates that a front will form on the upper boundary when there is a negatively curved density profile $\left(\partial^{2} \rho / \partial x^{2}<0\right)$, and that the estimate (3.7) of the timescale for frontogenesis is correct. These observations should remain true despite the unrealistic features of the model, viz. the horizontally infinite extent of the region of uniform curvature and the neglect of diffusive effects. We shall discuss first how diffusion can be included in the present model, and then compare the results obtained above with observations of fronts brought about by the presence of the $4^{\circ} \mathrm{C}$ density maximum of water in lakes and laboratory tanks (i.e. in finite regions).

In the non-diffusive model $\omega \propto x$, so that horizontal diffusion of vorticity $\left(\nu \partial^{2} \omega / \partial x^{2}\right.$ in (2.6)) has no effect. Thus the introduction of viscous effects into (3.18) does not alter the important feature that each field variable retains its particular form of $x$ dependence at all times. The only modification required is the inclusion of a term describing vertical diffusion of vorticity, so that (3.18) becomes

$$
\frac{\partial \Omega}{\partial \tau}-U \Omega+W \frac{\partial \Omega}{\partial \zeta}-\frac{1}{G r^{\frac{1}{2}}} \frac{\partial^{2} \Omega}{\partial \zeta^{2}}=R
$$




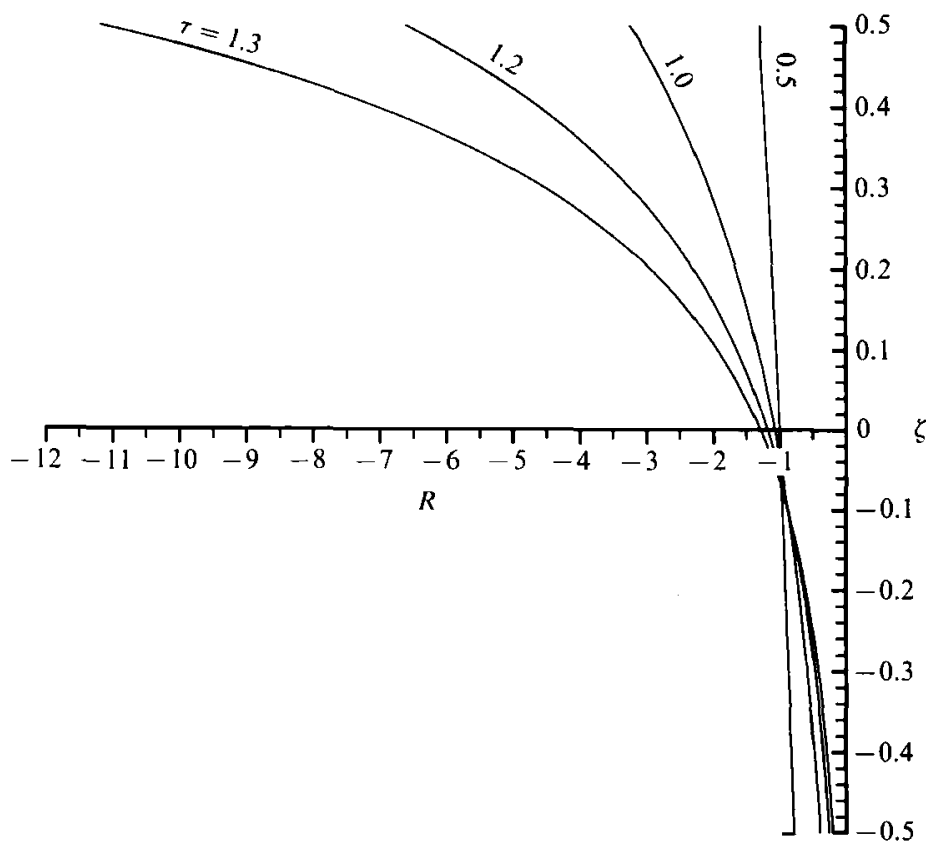

Figure 10. Vertical profiles of $R$ at dimensionless times $\tau=0.5,1.0,1.2,1.3$.

here, the Grashof number is defined as

$$
G r \equiv \frac{g \gamma h^{5}}{\bar{\rho} \nu^{2}} .
$$

Equation (3.20) could be integrated numerically (together with a temperature or density equation, derived below) using standard mthods applicable to parabolic equations if zero-stress boundary conditions were assumed, i.e.

$$
\Omega=0 \quad \text { at } \quad \zeta= \pm 0.5
$$

at each time-step an integration of $\Omega$ twice with respect to $\zeta$ would yield $U$ and $W$, as was done in the non-diffusive case. The adoption of no-slip boundary conditions

$$
U=0 \quad \text { at } \quad \zeta= \pm 0.5
$$

would be likely to pose greater difficulties.

Care needs to be taken in introducing diffusion to the density equation: in the context of a nonlinear vertical density profile, Matthews (1988) has pointed out that if the nonlinearity derives from the temperature (or salinity) profile there may be different dynamical consequences to the case where the nonlinearity is in the equation of state. The present study has been motivated by the latter case, with an equation of state

$$
\rho-\bar{\rho}=-\alpha(T-\bar{T})^{2},
$$

where $\bar{T}=4{ }^{\circ} \mathrm{C}$. Ignoring diffusion, it has been shown that the density profile remains quadratic at all times; hence the temperature profile remains linear, and horizontal diffusion of heat can have no effect on it. By analogy with (3.12), we therefore introduce $T^{*}(z, t)$ by

$$
T-\bar{T}=x T^{*}
$$


and then define the dimensionless temperature gradient $\Theta$ by

$$
\Theta=\frac{T^{*}}{\Gamma}
$$

where $\Gamma$ is the temperature gradient at time $t=0$ (so that $\gamma=\alpha \Gamma^{2}$ ). Instead of a density equation, we use the temperature equation $(2.5)$, which in dimensionless form becomes

$$
\frac{\partial \Theta}{\partial \tau}-U \Theta+W \frac{\partial \Theta}{\partial \zeta}-\frac{1}{\operatorname{Pr} G r^{\frac{1}{2}}} \frac{\partial^{2} \Theta}{\partial \zeta^{2}}=0
$$

where the Prandtl number is the ratio of diffusivities of vorticity and heat,

$$
\operatorname{Pr}=\nu / \kappa
$$

we may also substitute $R=2 \Theta^{2}$ in (3.20).

Alternatively, one could conceive of the quadratic density profile (3.1) being set up around a spatial maximum of salinity or minimum of temperature (away from $4^{\circ} \mathrm{C}$ if in water, so that the equation of state is assumed linear). However, a quadratic temperature profile implies that $\partial^{2} T / \partial x^{2}$ is independent of $x$, so although horizontal diffusion may affect the temperature, it will do so uniformly; the flow, which depends on horizontal temperature gradients, will be unaffected. Thus instead of (3.12) we write

$$
\rho-\bar{\rho}=-\frac{1}{2}\left\{x^{2} \rho^{*}(z, t)+\hat{\rho}(z, t)\right\}
$$

where $\hat{\rho}$ accounts for the effect of horizontal diffusion of heat. Substitutions of (3.24), together with (3.13)-(3.15), into the vorticity and density equations (including diffusive terms) yields a set of three evolution equations (for $w^{*}, \rho^{*}$ and $\hat{\rho}$ ). However, $\hat{\rho}$ does not appear in the equations for $w^{*}$ or $\rho^{*}$, indicating that horizontal diffusion again plays no part in the dynamics of frontogenesis.

The experiments of SL suggest that the sort of rapidly accelerating flow which we are considering is essentially inviscid, with any diffusive effects being confined to thin boundary layers on the upper and lower surfaces. Furthermore, (3.20) and (3.23) suggest that the thickness of such boundary layers will only grow to $O\left(G r^{-\frac{1}{4}}\right.$ ) (as a proportion of the total depth of fluid) within the timescale for frontogenesis $(\tau=O(1)) ; G r$ is expected to be large because of the generally small values of diffusivities. Since horizontal diffusion has been shown to have zero influence and vertical diffusion negliglble influence on frontogenesis with a quadratic density profile, it is therefore not worthwhile to pursue the solution of (3.20) and (3.23).

\subsection{Applications to cabbeling in lakes}

Cabbeling, i.e. the mixing of two water masses to form a mixture denser than either original body (Foster 1972), is well known from lakes in cold climates where the temperature range spans $4{ }^{\circ} \mathrm{C}$ during the spring warming period. The model described above is not directly applicable to such flows: the initial condition of a stationary body of fluid is unlikely ever to occur, and more importantly the idealization of a quadratic density profile of effectively infinite horizontal extent, and the consequent elimination of any role for horizontal Fickian mixing, is unrealistic. In reality, diffusive processes will allow a steady state to be reached, and it is this steady state which is observed in lakes with temperatures ranging either side of $4{ }^{\circ} \mathrm{C}$. Nevertheless, the idealized flow analysed above does have certain features in common with those observed in such lakes and in laboratory experiments on flows in water near $4^{\circ} \mathrm{C}$. 


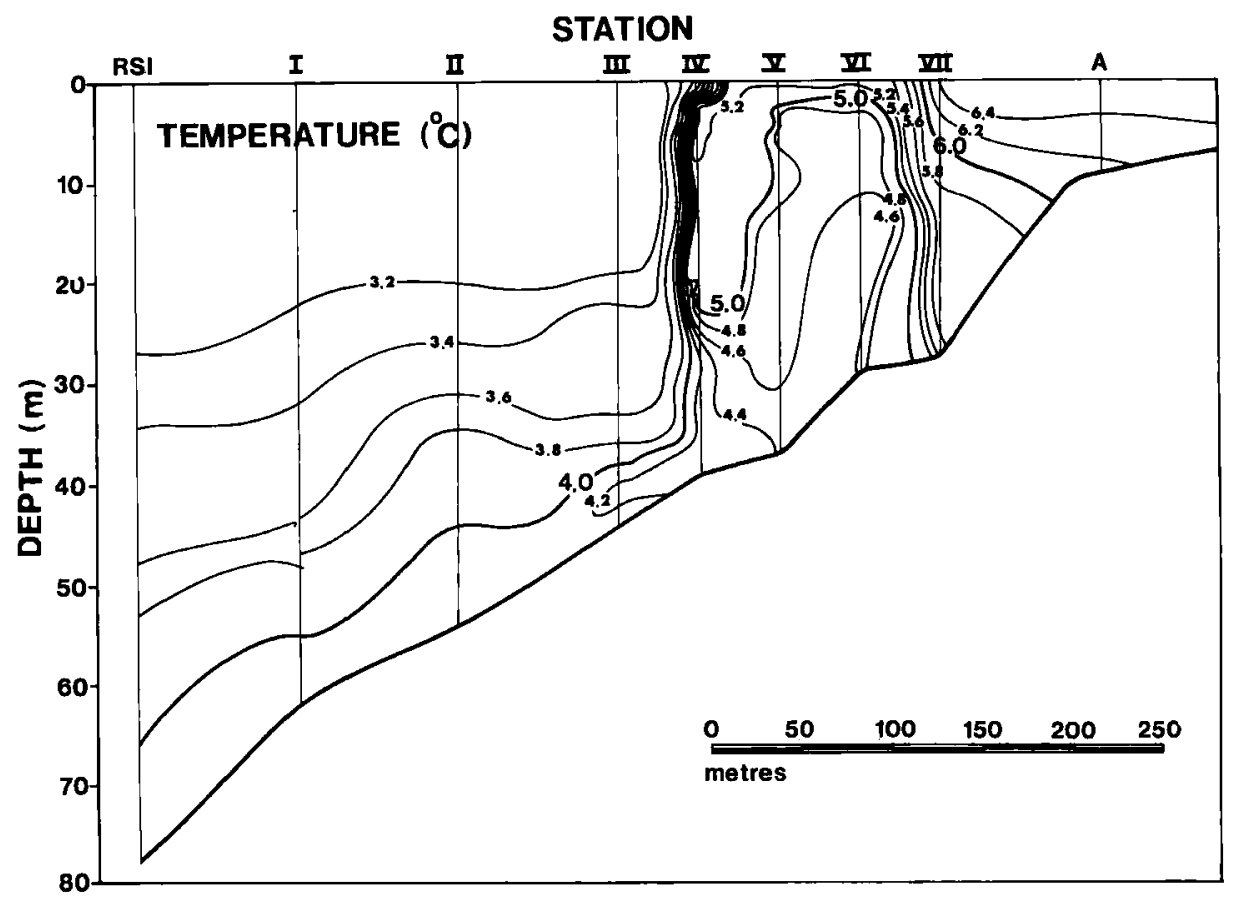

Figure 11. Isotherms on a cross-section of Kamloops Lake near the Thompson River inflow on 25 April 1975 (from Carmack 1979).

During the Spring warming period, the shallower parts of a lake will warm up faster than the deeper regions, so that there will be a period during which the deeper water will be cooler than $4{ }^{\circ} \mathrm{C}$ while the shallower water is warmer than $4{ }^{\circ} \mathrm{C}$. This situation has been studied in particular in the Great Lakes of North America (e.g. Elliott 1971 ; Hubbard \& Spain 1973); although the $4{ }^{\circ} \mathrm{C}$ isotherm gradually migrates from the shore to the centre of the lake, this is on a slow timescale compared to that of frontogenesis. Alternatively, a river which has warmed above $4{ }^{\circ} \mathrm{C}$ may flow into a lake still below $4^{\circ} \mathrm{C}$ (Carmack 1979). In either case, the phenomenon known as a thermal bar occurs: this is a front, centred at the $4{ }^{\circ} \mathrm{C}$ isotherm and oriented vertically, across which there is a rapid variation of temperature from, say, $3^{\circ} \mathrm{C}$ to $5{ }^{\circ} \mathrm{C}$. Although this front is very sharp at the surface, the horizontal temperature gradient rapidly decreases as one descends below about half-depth; at the lake bed, there is a broad region where the temperature of the water is approximately uniform at $4^{\circ} \mathrm{C}$. The structure is clear in figure 11 (taken from Carmack 1979), which shows isotherms in Kamloops Lake (the bulk of which is at $3^{\circ} \mathrm{C}$ ) near the inflow of the Thompson River (at $6.4^{\circ} \mathrm{C}$ ), although there are complications due to the bed topography. A similar isotherm plot was obtained by Ivey \& Hamblin (1989) in laboratory experiments in which a tank of water had one endwall cooled to $0{ }^{\circ} \mathrm{C}$ and the opposite wall heated to $8^{\circ} \mathrm{C}$.

The thermal bar therefore displays features in common with our idealized flow, namely frontogenesis in the upper part of the body of water and slackening of gradients in the lower part. Although the extent of the quadratic density profile is confined to the frontal region in a thermal bar situation (so that the descending flow is also concentrated there, rather than being uniform in the horizontal coordinate), the horizontal flow is similar to that predicted in our model, even at some distance 


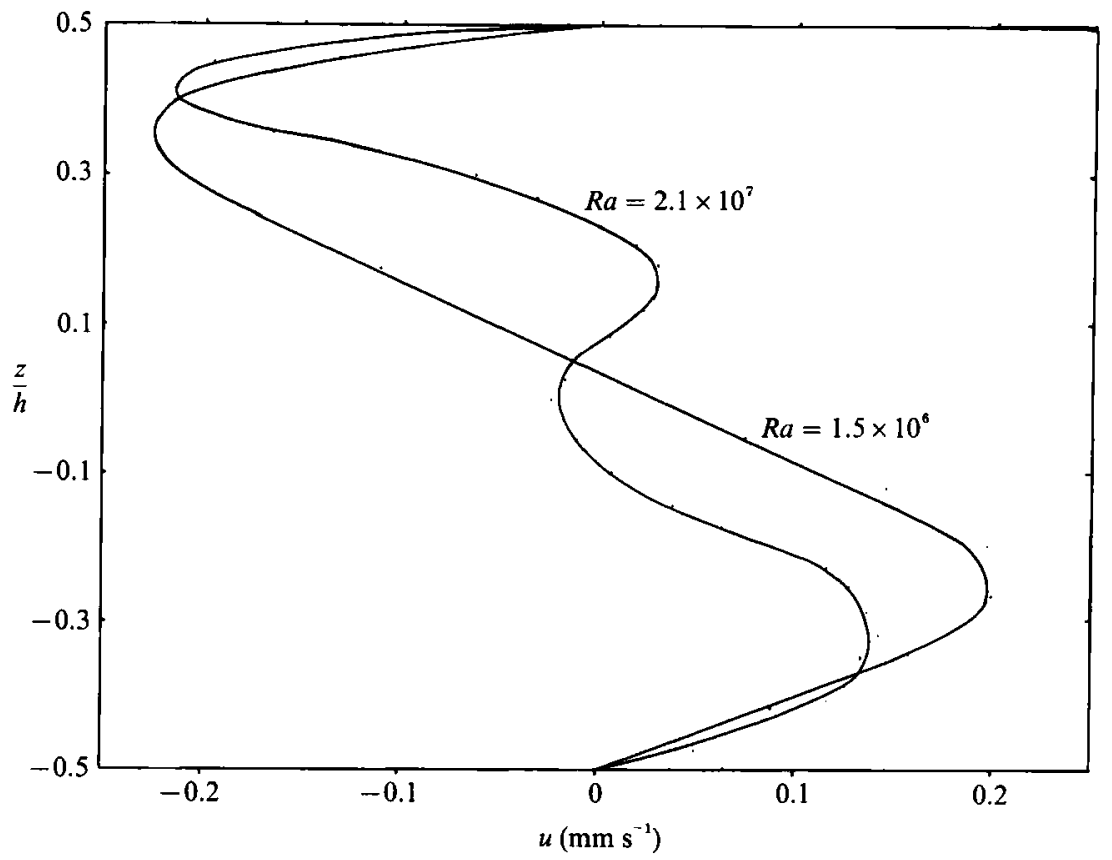

Figure 12. Profiles of horizontal velocity (interpolated from measurements plotted by Ivey \& Hamblin 1989) at a horizontal station between a thermal bar and a heated $\left(8^{\circ} \mathrm{C}\right)$ wall. Velocities are positive in the direction from the thermal bar towards the wall.

from the front. Figure 12 shows profiles of horizontal velocity measured by Ivey \& Hamblin (1989) about halfway between the thermal bar and the $8^{\circ} \mathrm{C}$ endwall, at two values of Rayleigh number $R a \equiv g \Delta \rho h^{3} / \bar{\rho} \nu \kappa$ (where $\Delta \rho$ is the density difference between water at $4{ }^{\circ} \mathrm{C}$ and at $8{ }^{\circ} \mathrm{C}$ ). At the lower $R a$, the profile is symmetric, with uniform shear (except in the boundary layers on the upper and lower surfaces), as in the $O(t)$ flow in our model (figure 2); at the higher $R a$, the flow is more like our $O\left(t^{3}\right)$ flow (if one ignores the boundary layers and the weak counterflows at mid-depth): there is a thin region of strong flow towards the $4{ }^{\circ} \mathrm{C}$ isotherm near the upper surface, and a broader region of weaker flow in the opposite direction below. Our model of course takes $R a=\infty$, but the development of that flow in time can be considered analogous to the effect of increasing $R a$ in the steady-state laboratory flow.

The thermal bar differs from a gravity-current front in that, because of the symmetry of the density profile, it does not propagate through the fluid. Even when the symmetry is broken, so that a propagating front might be expected to form, Carmack's (1979) results show that the thermal bar is dominant. The density difference between Kamloops Lake water at $3{ }^{\circ} \mathrm{C}$ and Thompson River water at $6.4^{\circ} \mathrm{C}$ is several times greater than that between the lake water and mixed water at $4{ }^{\circ} \mathrm{C}$; one might therefore expect to see a gravity current as in a lock-exchange flow, with river water moving lakeward above the denser lake water, but there is no sign in figure 11 of either water body penetrating beyond the thermal bar. Various factors may contribute to this : efficient turbulent mixing at the interface would favour the thermal bar rather than a gravity current; the rather steep bed topography of the lake would impede the motion of lake water towards the river mouth (and probably also plays an important role in creating the second front in the $5-6^{\circ} \mathrm{C}$ range). Furthermore, if the gravity current did start to develop, thereby tilting isopycnals to the horizontal, any mixing to produce $4{ }^{\circ} \mathrm{C}$ water at the interface would render the 
water column below the interface statically unstable. The resultant overturning in this lower layer would result in it becoming well-mixed at $4{ }^{\circ} \mathrm{C}$ (Foster 1972), so forming a thermal bar. The arrest of a gravity current and its replacement by a thermal bar have been observed experimentally by Marmoush, Smith \& Hamblin (1984).

\section{Linear density gradient near an endwall}

In LS's experiments, the effect of turbulent stirring on a lock-exchange gravity current was to produce a region in the centre of the tank where the fluid was vertically well mixed and had an approximately linear horizontal density variation (figure 13, dashed line; see also LS's figure 9). As time progressed, this region spread out until the density profile was linear along the entire length of the tank, except near the endwalls where it levelled out to satisfy the zero-flux boundary condition $\partial \rho / \partial x=0$; this is shown as the solid line in figure 13. Both profiles in figure 13 include curved regions which may be the cause of the frontogenesis observed by LS when the turbulent stirring was switched off; we shall investigate this theoretically using the method of expansion in powers of time.

First, however, it is of interest to study the gravity current which evolves from a density profile which is linear all the way to the endwalls, i.e. ignoring the zero-flux boundary condition. In a horizontally unbounded region, a linear density gradient will produce an accelerating shear flow which will rotate all isopycnals at the same rate, i.e. with no tendency to frontogenesis (SL). However, if this flow is blocked by an endwall, the horizontal velocity must be convergent where it is towards the wall and divergent where it is away from the wall. Frontogenesis is associated with convergence, and so it appears that the blocking effect of an endwall may be sufficient for the formation of a front, with no requirement for diffusive effects to produce curvature in the density profile

Consider an initially linear density profile with no stratification:

$$
\rho_{0}=\bar{\rho}(1+\alpha x)
$$

(i.e. with a change of sign compared with SL's definition of $\alpha$ ). The fluid occupies a rectangular tank,

$$
-\frac{1}{2} h<z<\frac{1}{2} h, \quad 0<x<L,
$$

but with $L \gg h$ we calculate the flow as for a semi-infinite region $x>0$, since the flow near the wall at $x=0$ will not be significantly affected by the far endwall. (In the finite tank the centro-symmetry property allows conditions near $x=L$ to be immediately deduced from those near $x=0$, as in Cormack, Leal \& Imberger's (1974) analysis : the points $(x, z)$ and $(L-x,-z)$ have equal and opposite flow velocities and equal denisty gradients.)

Substituting (2.7) and (4.1) into (2.2), the initial rate of vorticity generation is

$$
\omega_{1}=\frac{g}{\bar{\rho}} \frac{\partial \rho_{0}}{\partial x}=g \alpha
$$

and solving (2.4) yields

$$
\psi_{1}=\frac{g \alpha}{2}\left\{\frac{h^{2}}{4}-z^{2}-\frac{8 h^{2}}{\pi^{3}} \sum_{n=0}^{\infty} \frac{(-1)^{n} \exp \left(-\frac{(2 n+1) \pi x}{h}\right) \cos \frac{(2 n+1) \pi z}{h}}{(2 n+1)^{3}}\right\}
$$



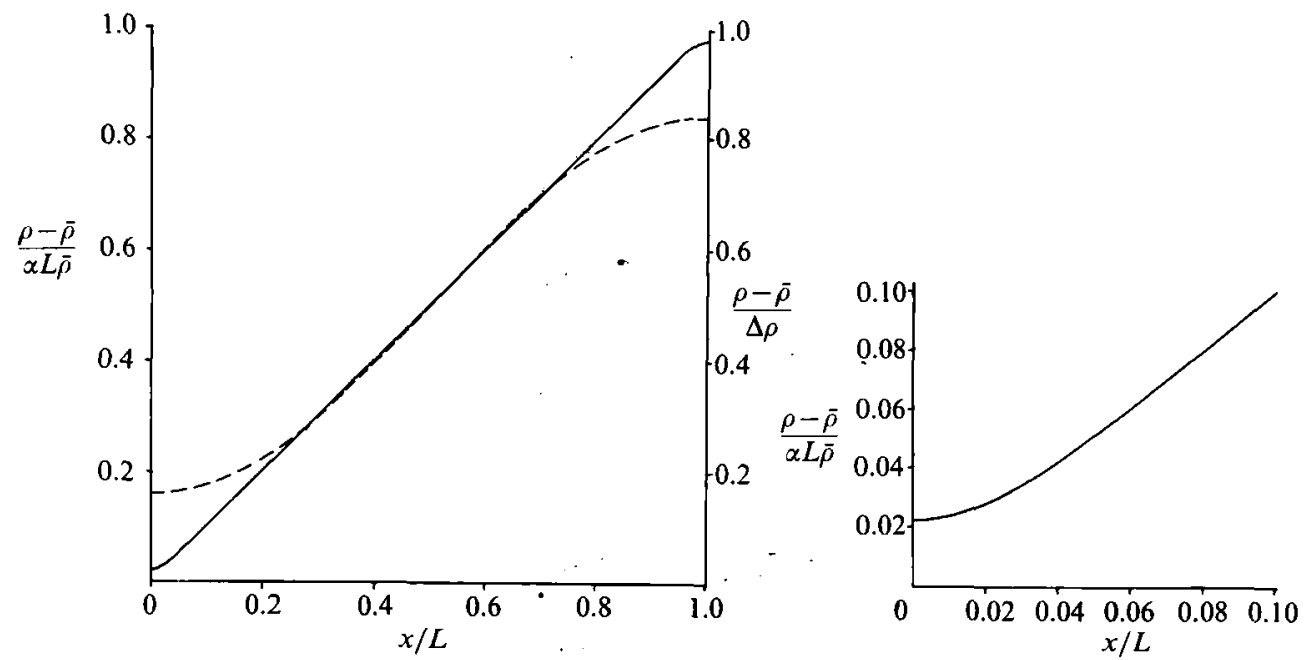

FIGURe 13. Horizontal density profiles used as initial conditions for calculations simulating LS's experiments : - , derived from the action of diffusion on an initially linear density profile with gradient $\alpha \bar{\rho}$ (with region near left-hand endwall shown on a larger scale in right-hand plot); -..-, derived from the action of Fickian shear dispersion on an initial step profile (step height $\Delta \rho$ at $x=0.5 L)$.

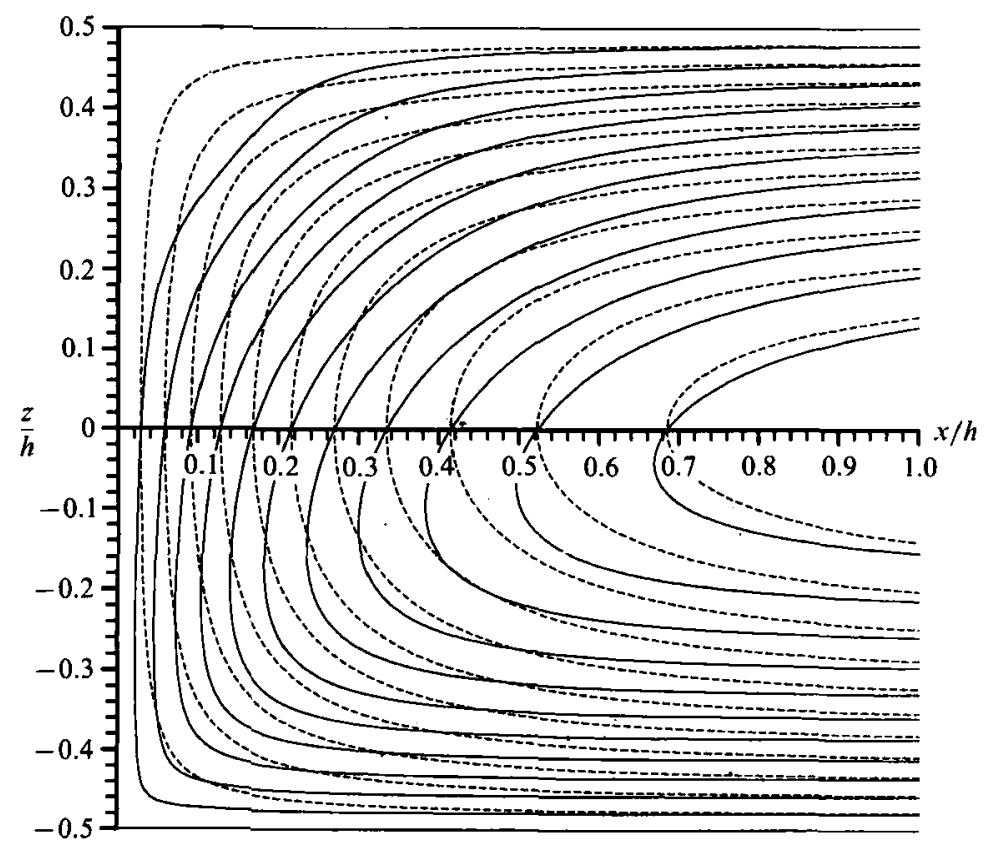

Figure 14. Streamlines in region near left-hand endwall at time $t=(g \alpha)^{-\frac{1}{2}}$ for flow driven by a linear density profile: -....., $O(t)$ flow only;,$- O(t)$ flow plus $O\left(t^{3}\right)$ perturbation.

The first two terms in the braces represent an unimpeded gravity current, and the Fourier series represents the blocking of this flow by the endwall, the effect of which is only felt within distances of order $h$ from the wall. The dashed lines in figure 14 are streamlines plotted using (4.3); it is clear from this diagram (or from differentiation of (4.3)) that the horizontal flow towards the wall in $z<0$ is convergent $(\partial u / \partial x<0)$ 
while the flow away from the wall in $z>0$ is divergent. There is also a vertical flow component, due entirely to blocking of the horizontal flow by the wall; the maximum vertical velocity is at the wall. When this upward flow meets the ceiling it will be turned back towards the centre of the tank, becoming the 'disturbance produced by reflection at the end of the channel' seen in SL's experiments (though this cannot be verified analytically using the expansion in powers of $t$ ).

The first-order flow perturbs the density profile at $O\left(t^{2}\right)$ :

$$
\rho_{2}=-\frac{1}{2} u_{1} \frac{\partial \rho_{0}}{\partial x}=\frac{g \alpha^{2} \bar{\rho}}{2}\left(-z+\frac{4 h}{\pi^{2}} \sum_{n=0}^{\infty} \frac{(-1)^{n}}{(2 n+1)^{2}} \exp \left(-\frac{(2 n+1) \pi x}{h}\right) \sin \frac{(2 n+1) \pi z}{h}\right) .
$$

In this expression, $-\frac{1}{2} g \alpha^{2} \bar{\rho} z$ represents the effects of an unimpeded gravity current: a uniform stable stratification with no change to horizontal density gradients. The effects of the endwall are a reduction in the stratification (because of the slowing down of the shear flow), while the horizontal gradient is steepened in $z<0$ and flattened in $z>0$. (In fact $\partial \rho_{2} / \partial x$ is singular at the lower corner of the wall, but one should be circumspect about interpreting this as a front; the density gradient varies strongly with $z$, and is in fact also singular at the upper corner, but there the 'front' faces the wrong way!)

Isopycnals are plotted for times $t=0$ and $t=(g \alpha)^{-\frac{1}{2}}$ in figure 15, using

$$
\rho=\rho_{0}+\rho_{2} t^{2}
$$

with (4.1) and (4.4); away from the wall, they are rotated uniformly, but nearer the wall the angle of rotation is reduced (indicating reduced stratification) and the spacing is changed (indicating steepening or flattening of the horizontal gradient). The S-shape is because the local angle of rotation depends on the local shear, and the distance from which the flow 'sees' the endwall is shorter for fluid near the horizontal boundaries than for fluid near $z=0$ (see figure 14).

Although frontogenesis appears to be occurring in the lower part of the tank, the front would appear at the wall (where the density gradient undergoes the greatest steepening) with the flow behind it tending to hold it against the wall. Thus no front would be expected to propagate along the tank; this accords with SL's observation that no front appeared when the initial density profile was linear.

It is nevertheless of interest to calculate the flow at $O\left(t^{3}\right)$. Unlike $\omega_{1}$, which is generated as a result of the initial linear density variation, the vorticity generation at $O\left(t^{3}\right)$ is entirely due to endwall blocking:

$$
\omega_{3}=\frac{1}{3} \frac{g}{\bar{\rho}} \frac{\partial \rho_{2}}{\partial x}=-\frac{2 g^{2} \alpha^{2}}{3 \pi} \sum_{n=0}^{\infty} \frac{(-1)^{n}}{(2 n+1)} \exp \left\{-\frac{(2 n+1) \pi x}{h}\right\} \sin \frac{(2 n+1) \pi z}{h} .
$$

This represents an increase in vorticity production where the density gradient is steepened at $O\left(t^{2}\right)$ and a decrease where it is flattened. The stream function at $O\left(t^{3}\right)$ is

$$
\begin{aligned}
\psi_{3}=\frac{g^{2} \alpha^{2}}{3 \pi}\left[\left(\frac{h^{2}}{4}-z^{2}\right) \sum_{n=0}^{\infty} \frac{(-1)^{n}}{2 n+1} \exp \{\right. & \left.-\frac{(2 n+1) \pi x}{h}\right\} \sin \frac{(2 n+1) \pi z}{h} \\
& \left.+h^{2} \sum_{n=1}^{\infty} A_{n} \sin \frac{2 n \pi z}{h} \exp \left\{-\frac{2 n \pi x}{h}\right\}\right]
\end{aligned}
$$

where

$$
A_{n}=-\frac{4}{\pi^{3}} \sum_{m=0}^{\infty} \frac{(-1)^{n}}{2 m+1}\left(\frac{1}{(2 n+2 m+1)^{3}}+\frac{1}{(2 n-2 m-1)^{3}}\right) \text {. }
$$




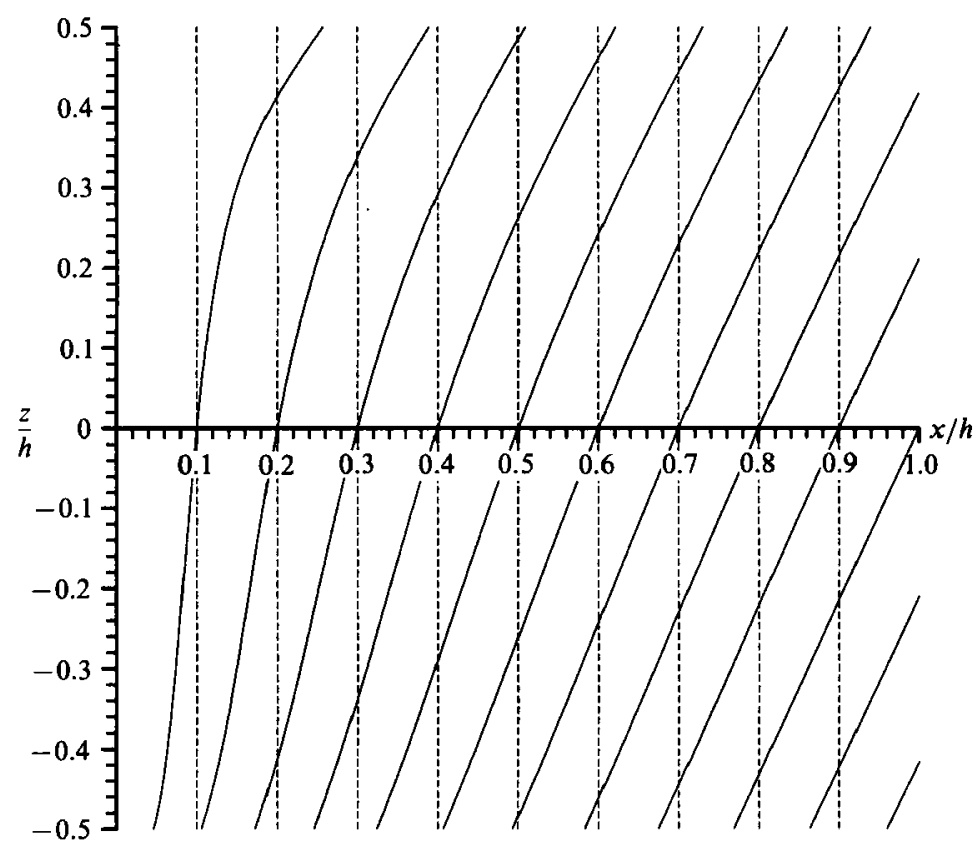

Fiaure 15. Isopycnals in region near left-hand endwall at times $t=0$ (dashed lines) and $t=(g \alpha)^{-\frac{1}{8}}$ (solid lines) for flow driven by a linear density profile. Density calculated as $\rho=\rho_{0}+\rho_{2} t^{2}$, using (4.1) and (4.4).

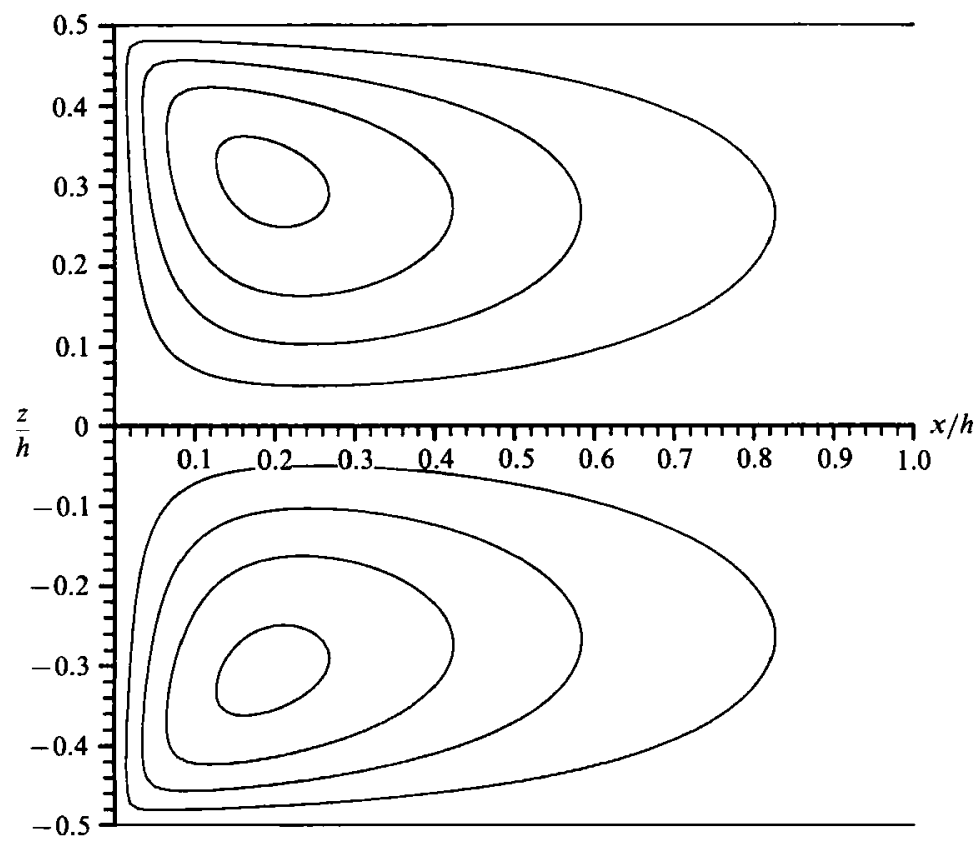

FIGURE 16. Streamlines in region near left-hand endwall for $O\left(t^{3}\right)$ flow perturbation in flow driven by a linear density profile.

The first Fourier series in (4.6) is the circulation resulting directly from the $O\left(t^{2}\right)$ density gradient changes, and the second series shows how this circulation is itself modified by the presence of the endwall. Streamlines for $\psi_{3}$ are plotted in figure 16, which shows a circulation confined to a region of horizontal extent $\sim h$ near the 


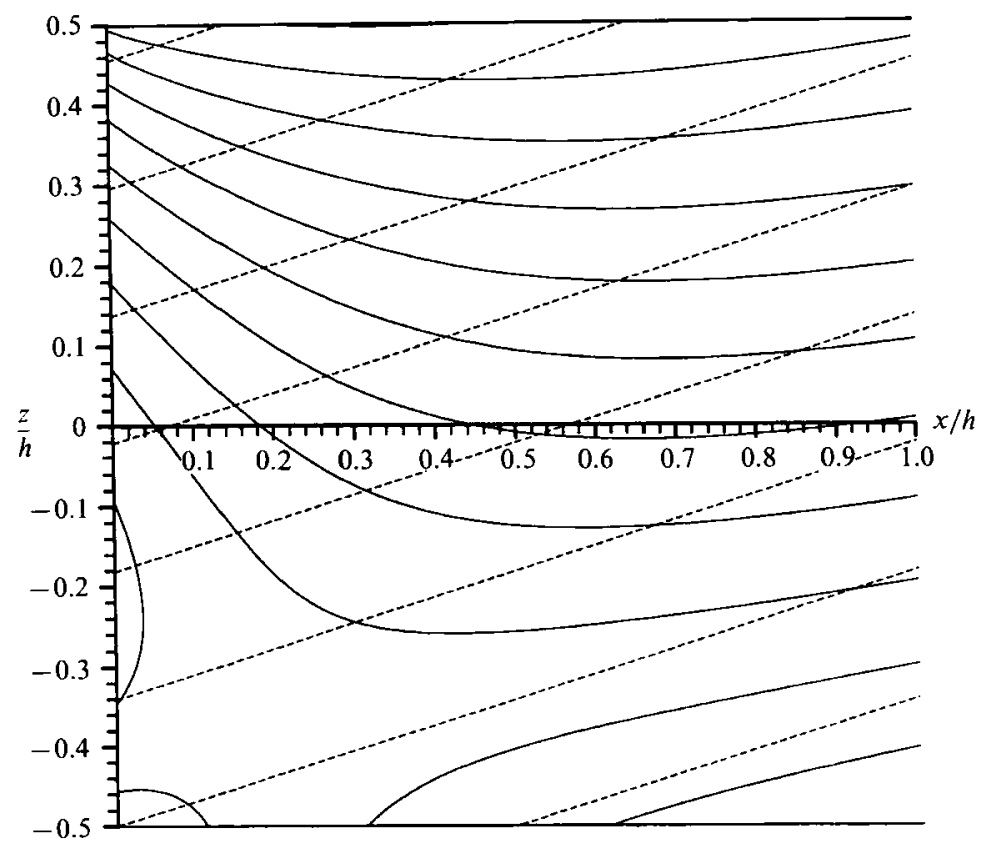

Figure 17. Isopycnals in region near left-hand endwall for flow driven by a linear density profile with initial stratification $\beta=\pi \alpha$, at time $t=0$ (dashed lines) and $t=2(g \alpha)^{-\frac{1}{2}}$ (solid lines).

endwall. This circulation perturbs the $O(t)$ flow, and the resultant flow (represented by $\left.\psi=\psi_{1} t+\psi_{3} t^{3}\right)$ is shown at time $t=(g \alpha)^{-\frac{1}{2}}$ by the solid streamlines in figure 14 . This shows similar features to figure 3 (but upside-down): the flow towards the wall is stronger, and confined to a smaller portion of the height of the tank, than the flow away from the wall; the vertical velocity is also greater in the lower half of the tank.

\subsection{Effects of initial stable stratification}

To simulate SL's experiment with a linear density profile more precisely, it is necessary to take account of the initial stable stratification. SL's initial condition on the density was

$$
\rho_{0}=\bar{\rho}(1+\alpha x-\beta z)
$$

with $\beta / \alpha=\pi$, and we shall calculate the subsequent flow and density perturbations as before.

The flow at $O(t)$ is unaffected by $\beta$, and is still given by (4.2) and (4.3). However, the $O(t)$ vertical flow advects dense fluid upwards, so that the $O\left(t^{2}\right)$ density perturbation (4.4) is modified to

$$
\begin{aligned}
\rho_{2}=-\frac{1}{2}\left(u_{1} \frac{\partial \rho_{0}}{\partial x}+w_{1} \frac{\partial \rho_{0}}{\partial z}\right)=\frac{g \alpha^{2} \bar{\rho}}{2}(- & +\frac{4 h}{\pi^{2}} \sum_{n=0}^{\infty} \frac{(-1)^{n}}{(2 n+1)^{2}} \exp \left\{-\frac{(2 n+1) \pi x}{h}\right\} \\
& \left.\times\left(\sin \frac{(2 n+1) \pi z}{h}-\frac{\beta}{\alpha} \cos \frac{(2 n+1) \pi z}{h}\right)\right)
\end{aligned}
$$

in which the extra term involving $\beta$ reduces the density gradient everywhere. The effects of this can be seen in figure 17, in which isopycnals (for $\rho=\rho_{0}+\rho_{2} t^{2}$ ) are plotted at times $t=0$ and $t=2(g \alpha)^{-\frac{1}{2}}$ with $\beta / \alpha=\pi$. Only in a shallow layer at the base of the tank is the horizontal gradient steepened (whereas steepening occurred in the entire lower half of the $\operatorname{tank}$ with $\beta=0$ ); elsewhere it is flattened or even reversed 
(where the isopycnals are recurved upwards towards the endwall, a feature clearly visible in SL's figure 5). This may be explained as follows: when vertical flow is taken into account, there are additional terms

$$
-w \frac{\partial^{2} \rho}{\partial z \partial x}-\frac{\partial w}{\partial x} \frac{\partial \rho}{\partial z}
$$

on the right-hand side of equation (1.2) for the rate of change of density gradient. The second term in (4.9) (which acts at $O\left(t^{2}\right)$, whereas the first term only comes in at $O\left(t^{4}\right)$ ) shows that because the vertical velocity decreases with distance from the wall (see figure 14) dense fluid will be pushed upwards most rapidly at the low-density end of tank; hence the horizontal density gradient will be reduced or even made negative.

The reversal of the density gradient in a substantial region near the upper corner of the endwall implies a reversal in vorticity generation, suppressing the initial gravity-current flow near the wall. The dense fluid which is initially forced upwards when the gravity current is blocked by the endwall will tend to slump back down, presumably suppressing any subsequent reflected flow along the ceiling. One may speculate that if SL had conducted experiments with zero initial stratification they would have observed rather greater reflected disturbances than actually occurred.

\subsection{Effects of diffusion near an endwall}

In SL's experiments an initially linear density gradient was obtained by setting up a linear salinity gradient (the equation of state being linear). However, the diffusive zero-flux boundary condition implies an initial discontinuity in the salinity and density gradients at the endwalls; hence diffusion must be important near the endwalls at small times, even if the diffusivity is very small. This implies that there is some timespan $0<t<t_{\delta}$, before the gravity-driven motion becomes significant, during which diffusion is changing the density profile near the walls. After $t=t_{\delta}$, diffusion has negligible effect on the rapidly accelerating gravity current. The density profile which obtains after a period $t_{\delta}$ of pure diffusion can therefore be taken as the initial condition for the subsequent non-diffusive development of a gravity current (i.e. we ignore any transition period between the diffusive and gravity-current phases).

We may estimate the magnitude of $t_{\delta}$ as the time at which the gravity-driven flow (initially with acceleration $u_{1}$, starting from rest) 'overtakes' the diffusive smoothing of the density profile:

$$
\frac{1}{2} u_{1} t_{\delta}^{2}=2\left(\kappa t_{\delta}\right)^{\frac{1}{2}} \equiv \delta
$$

(i.e. defining $\delta$ as the width of the region adjacent to the endwall within which diffusive effects are felt at time $t_{\delta}$ ). We therefore obtain acceleration-diffusion timeand lengthscales (cf. Kay 1990)

From (4.3) we have

$$
\begin{aligned}
& t_{\delta}=\left(16 \kappa / u_{1}^{2}\right)^{\frac{1}{3}} \\
& \delta=\left(32 \kappa^{2} / u_{1}\right)^{\frac{1}{3}}
\end{aligned}
$$

$$
u_{1} \sim g \alpha h
$$

thus with $\kappa \sim 10^{-9} \mathrm{~m}^{2} \mathrm{~s}^{-1}$ (the molecular diffusivity for salt in water) and $\alpha \sim 10^{-2} \mathrm{~m}^{-1}, h \sim 10^{-1} \mathrm{~m}$ (as in SL's experiments), we find

$$
t_{\delta} \sim 10^{-1} \mathrm{~s}, \quad \delta \sim 10^{-5} \mathrm{~m}
$$

These values are so small that we are justified in ignoring any diffusive effects on the density profile when analysing SL's experiments; certainly none were observed. On 
the other hand, LS created a linear density profile by the action of turbulence on a lock-exchange gravity current, so in this case it would be more appropriate to take $\kappa$ as the eddy diffusivity which LS found to be of order $3 \times 10^{-4} \mathrm{~m}^{2} \mathrm{~s}^{-1}$. The value of $\delta$ is then about $0.05 \mathrm{~m}$, i.e. similar to the widths of the regions of curvature near the endwalls in the otherwise linear density profiles in LS's figure 9 .

During the purely diffusive phase $0<t<t_{\delta}$, the density profile evolves from the initial condition (4.1) according to

$$
\frac{\partial \rho}{\partial t}=\kappa \frac{\partial^{2} \rho}{\partial x^{2}}
$$

with endwall boundary conditions

$$
\frac{\partial \rho}{\partial x}=0 \quad \text { at } \quad x=0, x=L .
$$

Solving (4.15), we find

$$
\rho=\bar{\rho}\left\{1+\frac{1}{2} \alpha L-\frac{4 \alpha L}{\pi^{2}} \sum_{n=0}^{\infty} \frac{1}{(2 n+1)^{2}} \exp \left\{-\frac{(2 n+1)^{2} \pi^{2} \kappa t}{L^{2}}\right\} \cos \frac{(2 n+1) \pi x}{L}\right\} .
$$

(Solutions of (4.15) are not available in a convenient form for a semi-infinite tank as for the non-diffusive case; when plotting isopycnals and streamlines from the formulae derived below, we shall use an aspect ratio $h / L=0.1$, sufficient to prevent the far endwall having any noticeable influence on conditions in $0<x<h$.) The initial condition for the development of the gravity current is then obtained by substituting $t=t_{\delta}=\delta^{2} / 4 \kappa$ in $(4.17)$ :

$$
\rho_{0}=\bar{\rho}\left\{1+\frac{1}{2} \alpha L-\frac{4 \alpha L}{\pi^{2}} \sum_{n=0}^{\infty} \frac{1}{(2 n+1)^{2}} \exp \left\{-\frac{(2 n+1)^{2} \pi^{2} \delta^{2}}{4 L^{2}}\right\} \cos \frac{(2 n+1) \pi x}{L}\right\} ;
$$

henceforth $\delta$ is treated as a constant. The value $\delta=0.04 L$ (comparable to the widths of the regions of curvature in profiles measured at later times in LS's figure 9) was used in (4.18) to plot the initial density profile shown as the solid line in figure 13.

We can now proceed as in the non-diffusive case, using (4.18) to calculate the $O(t)$ vorticity from

$$
\omega_{1}=\frac{g}{\rho} \frac{\partial \rho_{0}}{\partial x}
$$

and then solving (2.4) for the stream function:

$$
\psi_{1}=\frac{4 g \alpha L^{2}}{\pi^{3}} \sum_{n=0}^{\infty} \frac{1}{(2 n+1)^{3}} \exp \left\{-\frac{(2 n+1)^{2} \pi^{2} \delta^{2}}{4 L^{2}}\right\}\left(1-\frac{\cosh \frac{(2 n+1) \pi z}{L}}{\cosh \frac{(2 n+1) \pi h}{2 L}}\right) \sin \frac{(2 n+1) \pi x}{L} .
$$

The solid lines in figure 18 are streamlines plotted using (4.19) with $\delta=0.04 L$, with streamlines for the non-diffusive case shown dashed for comparison.

Essentially, we can think of the effect of diffusion as giving the flow 'advanced warning' of the endwall. The diffusively reduced density gradient in $0<x \leqslant \delta$ causes the flow to already be slowed down before it feels the blocking effect of the endwall. Hence in figure 18, the horizontal velocity is everywhere less in the diffusive than the 


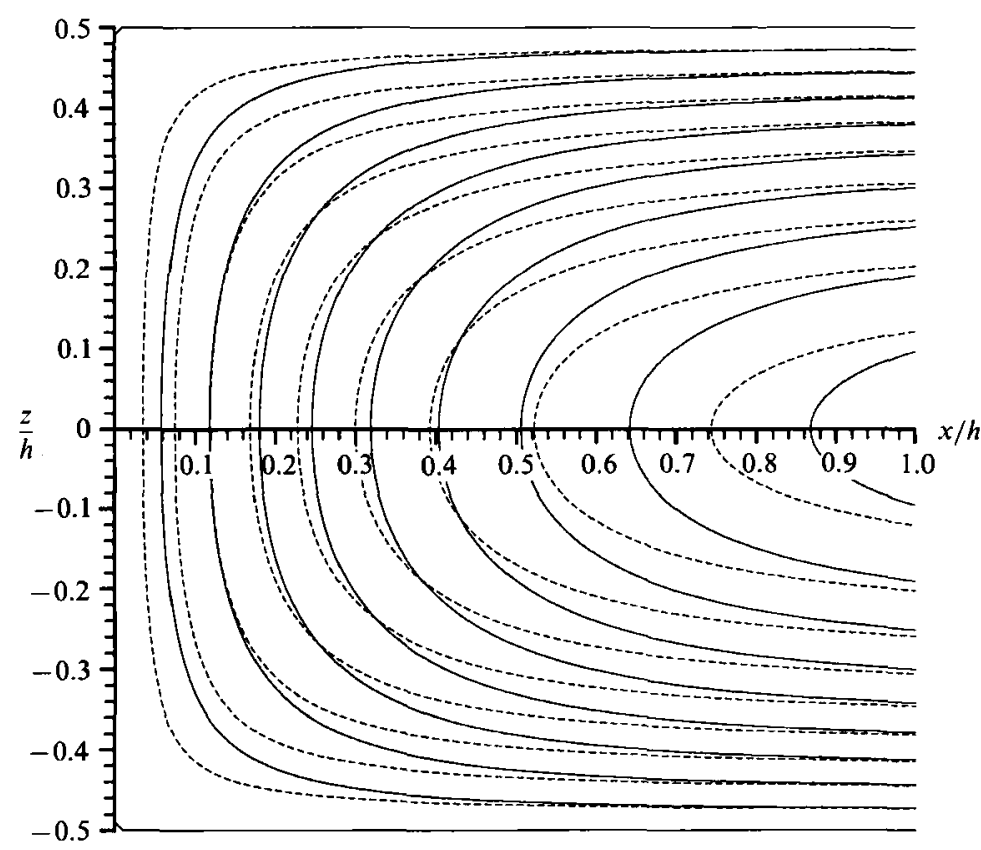

FiaURE 18. Streamlines for $O(t)$ flow near left-hand endwall : ...---, initial density profile strictly linear; $\_$, initial density profile as solid line in figure $\mathbf{1 3}$.

non-diffusive case. However, the flow far from the endwall must be as for an unimpeded gravity current in both cases; thus if diffusion reduces the intensity of convergence $|\partial u / \partial x|$ in $0<x<\delta$, there must be an increased convergence somewhere else. The vertical velocity on $z=0$ is $w=-\int_{-n / 2}^{0}(\partial u / \partial x) \mathrm{d} z$ and hence provides an indication of the convergence in the lower half of the tank at a given horizontal station; the spacing of streamlines crossing $z=0$ in figure 18 clearly indicates the vertical flow (and hence convergence) is reduced in $0<x<\delta$ but increased in $x>\delta$. Hence convergence, the cause of frontogenesis, is spread out over a greater horizontal distance by diffusion; it is therefore possible that a front might form at some distance (of order $\delta$ ) from the wall and then propagate towards the wall.

This effect may be seen in the isopycnal plots (figure 19a,b) in which (4.18) and (4.19) have been used to calculate the density perturbation

$$
\rho_{2}=-\frac{1}{2} u_{1} \frac{\partial \rho_{0}}{\partial x} \text {. }
$$

In figure $19(a)$ the isopycnals are plotted at the same times as in the non-diffusive case (figure 15), but to make the comparison clearer figure $19(b)$ shows isopleths of the perturbation $\rho_{2}$ only, for $\delta=0.04 L$ and $\delta=0$ (non-diffusive). Changes to the horizontal density gradient are most easily seen in figure $19(b)$ by examining the intersections of perturbation isopycnals with the horizontal boundaries: without diffusion, the changes are concentrated near the endwall, whereas they are spread out more evenly over a region $0<x \leqslant 2 \delta$ when diffusion is allowed to act. From figure $19(a)$, the steepest density gradient at time $t=(g \alpha)^{-\frac{1}{2}}$ is at the lower boundary at $x \approx \delta$ (rather than at the wall, where $\partial \rho_{2} / \partial x$ was singular in the non-diffusive case), so this is where frontogenesis might be expected to occur. (The singularity in the density gradient could also be removed from SL's results with a piecewise-linear density profile if the initial profile was smoothed by diffusion.) 
(a)

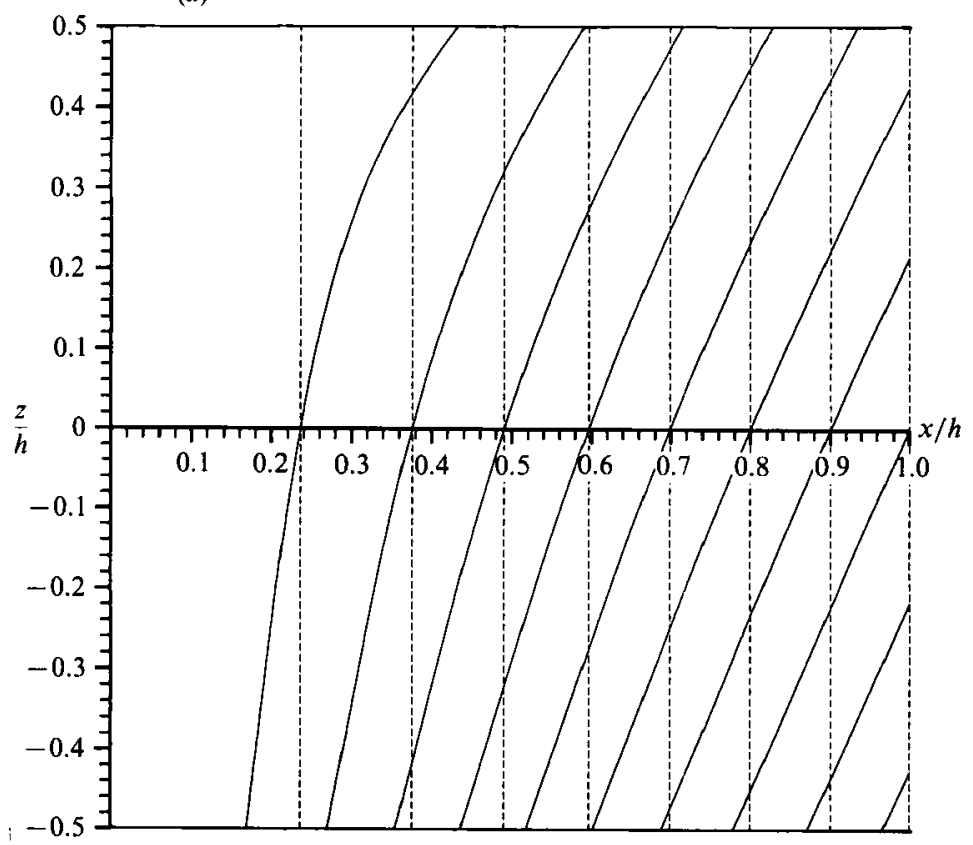

(b)

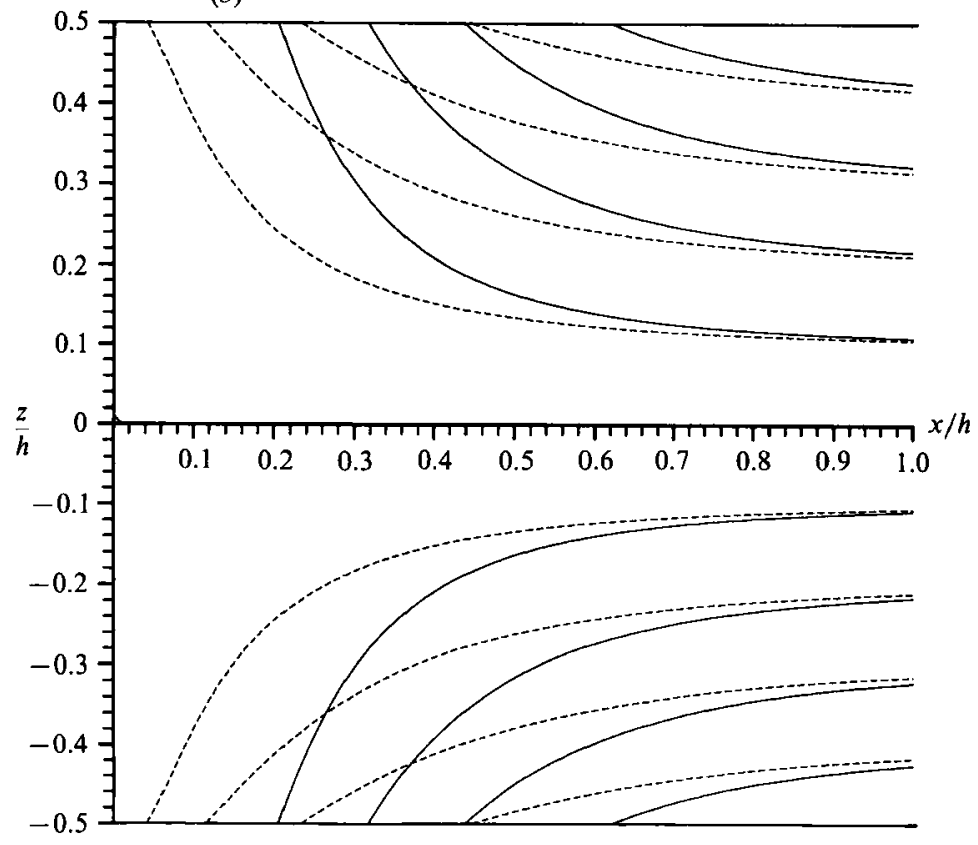

Fiaure 19. (a) Isopycnals in region near left-hand endwall at times $t=0$ (dashed lines) and $t=(g \alpha)^{-\frac{1}{2}}$ (solid lines) for flow driven by a density profile shown as solid line in figure 13 . (b) $O\left(t^{2}\right)$ density perturbation for flow near an endwall : --.---, initial density profile strictly linear; initial density profile as solid line in figure 13.

Diffusion also has the effect of spreading out the region in which vertical stratification is reduced by the slowing down of the gravity current. In the comparison between figures 15 and $19(a)$, this shows up in the reduction in the angle of rotation of isopycnals (at any given horizontal station) in the diffusive case. Note 
that the zero-flux condition is not satisfied at the horizontal boundaries (where the isopycnals should be vertical), since the model does not take account of diffusion after the gravity current is set into motion; SL's experiments show that boundarylayer effects are rapidly suppressed as the flow gathers momentum.

\subsection{Frontogenesis resulting from an initial density profile with significant curvature throughout}

The fronts which formed after the turbulence was switched off in LS's experiments were already fairly well-defined at a distance of $0.6 \mathrm{~m}$ from the endwalls (LS, figure 12), in contrast to the estimated value of $0.05 \mathrm{~m}$ for the distance $\delta$ at which frontogenesis is expected to take place according to the above analysis. Furthermore, the analysis predicts the frontogenesis to be weak, since figure $19(a)$ shows the maximum of density gradient at $x \approx \delta$ to be rather shallow. It therefore appears that curvature of the density profile produced by diffusive effects near the endwalls may not be sufficient to explain the fronts observed by LS. However, the turbulence may have been switched off before the density profile had become linear over all but a $\delta$ region adjacent to each endwall (P.F. Linden, personal communication); there would then be significant curvature over the entire length of the tank (as in the density profiles measured at earlier times in LS's figure 9), and frontogenesis may occur further from the walls.

The density profiles measured by LS were the result of an interaction between the buoyancy-driven shear flow and the turbulent mixing, producing a shear-dispersion effect (Taylor 1953). If this effect can be represented by a Fickian dispersion coefficient (as assumed by LS), these profiles will be solutions at various times $t$ of the diffusion equation (4.15) with the zero-flux boundary conditions (4.16), but evolving from an initial step profile

$$
\rho=\left\{\begin{array}{ll}
\bar{\rho}, & 0<x<\frac{1}{2} L \\
\bar{\rho}+\Delta \rho, & \frac{1}{2} L<x<L
\end{array}\right\} \text { at } t=0 .
$$

The required solution is

$$
\rho=\bar{\rho}+\frac{1}{2} \Delta \rho\left\{1-\frac{4}{\pi} \sum_{n=0}^{\infty} \frac{(-1)^{n}}{2 n+1} \exp \left\{-\frac{(2 n+1)^{2} \pi^{2} \kappa t}{L^{2}}\right\} \cos \frac{(2 n+1) \pi x}{L}\right\} .
$$

If the turbulence is switched off at a time $t_{\lambda}$, then the profile (4.22) which obtains at $t=t_{\lambda}$ will be the initial condition for the subsequent development of the gravity current. Introducing the lengthscale $\lambda=2\left(\kappa t_{\lambda}\right)^{\frac{1}{2}}$, this initial condition becomes

$$
\rho_{0}=\bar{\rho}+\frac{1}{2} \Delta \rho\left\{1-\frac{4}{\pi} \sum_{n=0}^{\infty} \frac{(-1)^{n}}{2 n+1} \exp \left\{-\frac{(2 n+1)^{2} \pi^{2} \lambda^{2}}{4 L^{2}}\right\} \cos \frac{(2 n+1) \pi x}{L}\right\},
$$

which is plotted for $\lambda=0.5 L$ as the dashed profile in figure 13 .

The analysis of the development of the gravity current from this initial profile proceeds along the same lines as that for a linear profile altered by diffusion near the endwalls (following (4.18)). The stream function at $O(t)$ is

$$
\psi_{1}=\frac{2 g L \Delta \rho}{\pi^{2} \bar{\rho}} \sum_{n=0}^{\infty} \frac{(-1)^{n}}{(2 n+1)^{2}} \exp \left\{-\frac{(2 n+1)^{2} \pi^{2} \lambda^{2}}{4 L^{2}}\right\}\left(1-\frac{\cosh \frac{(2 n+1) \pi z}{L}}{\cosh \frac{(2 n+1) \pi h}{2 L}}\right) \sin \frac{(2 n+1) \pi x}{L}
$$

and the density perturbation $\rho_{2}$ is calculated from (4.20), using (4.24) and (4.23) to obtain $u_{1}$ and $\partial \rho_{0} / \partial x$ respectively. Isopycnals (for $\rho=\rho_{0}+\rho_{2} t^{2}$ ) are plotted in figure 


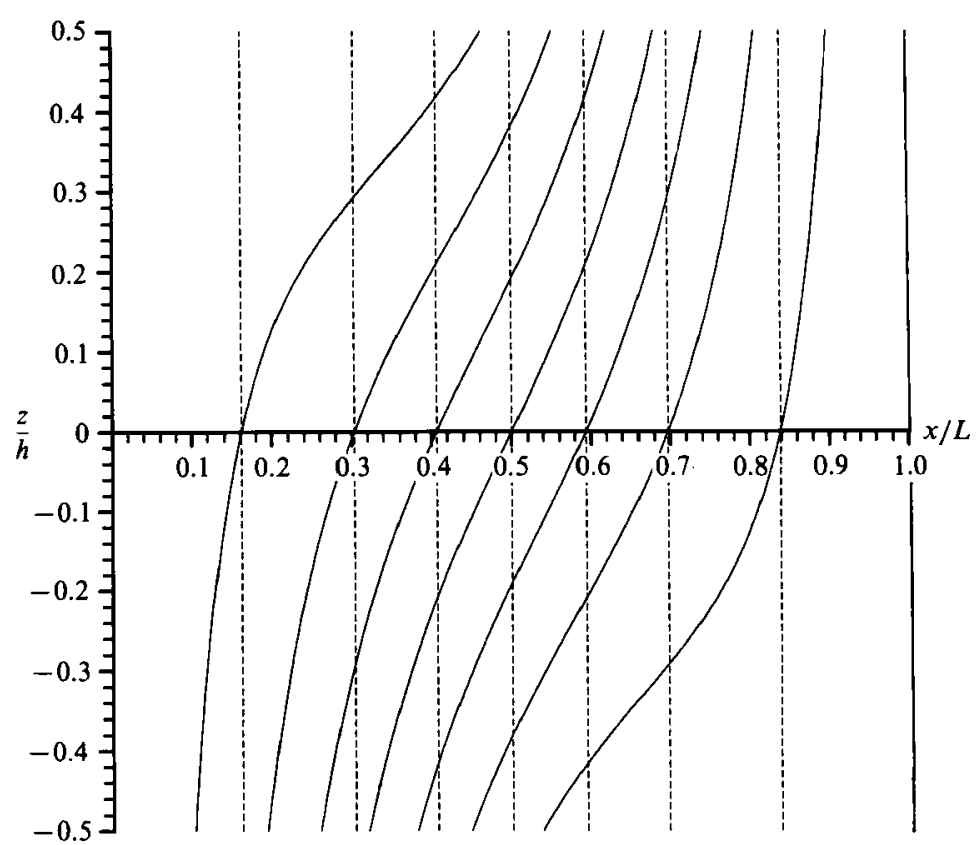

Figure 20. Isopycnals at times $t=0$ (dashed lines) and $t=3(g \Delta \rho / L \bar{\rho})^{-\frac{1}{2}}$ for flow driven by the initial density profile shown as dashed line in figure 13. Note that (unlike previous isopycnal plots) this shows the entire length of the tank, with a vertical scale exaggeration by a factor $L / h$.

20 , taking $\lambda=0.5 L$; this plot is for time $t=3(g \Delta \rho / L \bar{\rho})^{-\frac{1}{2}}$ because the changes to the density gradient are not very clearly visible at $t=(g \Delta \rho / L \bar{\rho})^{-\frac{1}{2}}-$ in reality the positive feedback effect would enhance the steepening of the gradient on the timescale $(g \Delta \rho / L \bar{\rho})^{-\frac{1}{2}}$, whereas we have simply exaggerated the size of the perturbation $\rho_{2}$, without altering its location, by plotting the $O\left(t^{2}\right)$ density at a larger time. Figure 20 shows the steepest density gradients to be on the upper and lower boundaries, in flow moving towards the endwalls at a distance of about $0.3 L$ from the walls; this is the region where frontogenesis occurred in the experiment recorded in LS's figure 12. Comparing with the initial density profile (figure 13), frontogenesis is seen to occur where the flow is just entering a region of curvature, rather than where the curvature is greatest. The term

$$
\frac{\partial u}{\partial x} \frac{\partial \rho}{\partial x}
$$

in (1.2) shows that the rate of steepening of the density gradient is proportional to convergence (resulting from curvature) and to the existing gradient. Thus the most favourable location for frontogenesis will in general be somewhere between a maximum of curvature and an inflexion point (maximum gradient but zero curvature) in the density profile.

\subsection{Comparison with other gravity-driven flows in shallow cavities}

Our analysis has been intended to model flows which evolve too rapidly for diffusive transfer of heat, salt or momentum to play a significant role. Thus the temperature or salinity distribution is 'frozen' into the fluid and changes only as a result of variations in the advective velocity within the fluid. However, to model some geophysical situations it is more appropriate to consider slower flows in which 
diffusion of heat or salt plays a primary role. Relevant examples are the flow in a shallow rectangular cavity with differentially heated endwalls (Cormack et al. 1974; Patterson \& Imberger 1980) or with non-uniform internal heating (Patterson 1984).

The thermal forcing specified by these authors ensures that the steady state will involve a circulation of fluid, whereas without heat input (as in our study), the fluid must eventually become stationary with horizontal isopycnals. However, even where the evolution of the flow from an initially stationary state is considered (by Patterson \& Imberger 1980 and Patterson 1984), there are only limited similarities to our results. (The steepening of the temperature gradient near the endwalls found by Patterson is by a completely different mechanism to that of flow convergence described above.) In fact, the greatest similarities to our results are in the steadystate analysis of Cormack et al. (1974), for two reasons. First, these authors showed that in the steady state the temperature varies linearly between the temperatures of the heated endwalls (to first order), so that the lowest-order forcing will be identical to the case of a linear density profile analysed above; secondly, they performed a perturbation expansion in the aspect ratio $h / L$, which, like our expansion in time, decomposes the complicated interaction between flow and density profile into a series of stages.

The most obvious similarity between Cormack et al.'s (1974) results and ours (with a linear profile) is the division of the cavity into a core region with a parallel shear flow and end regions (of width $\sim h$ ) in which the flow is turned round. Looking in more detail, the similarities between the stages in the two problems can be seen in Cormack et al.'s (1974) figure 4 (isopleths of $\theta_{2}^{\prime}$, comparable to $\rho_{2}$ in our figure $19 b$ ) and figure $5\left(\psi_{1}^{\prime}\right.$, comparable to $\psi_{3}$ in our figure 16); however, the analogy suggests that their attribution of the skewness in the streamlines at first order to vertical stratification retarding downward flow may not be correct; it is instead a result of the changes to the density gradient in the end regions wrought by the zero-order flow.

\section{Summary and conclusions}

A gravity current driven by a nonlinear horizontal density variation will develop a front by a mechanism first identified by SL and investigated further here. The nonuniform forcing of the flow implies that its horizontal component must be convergent in some region, and this convergence brings vertical isopycnals closer together. This steepening of the density gradient in turn drives the convergent flow more strongly, so there is a positive feedback which causes a singularity in density gradient (i.e. a front) to appear on a horizontal boundary of the fluid in finite time. Frontogenesis occurs near the upper boundary (and the density gradient is slackened near the lower boundary) if the density profile is negatively curved, and vice versa.

Negative curvature is present in a body of fresh water with a temperature profile straddling the temperature of maximum density; the 'thermal bar' which appears in such cases is a front which is well-defined only in the upper half of the water body. The analysis with a uniformly curved density profile of infinite horizontal extent showed this general feature, but was unable to predict the horizontal station at which a front would form. A more realistic model was also analysed, in which the fluid was confined between endwalls with the density gradient reaching a maximum in the middle of the container and falling to zero at the walls: this study showed that frontogenesis would occur where the flow was just entering a region of significant curvature after passing through a maximum in the density gradient. Such a profile could be produced by the requirement to satisfy the diffusive zero-flux boundary 
condition at a wall in an otherwise linear density profile, but in this case the region of curvature near the wall would be rather narrow and it is doubtful whether a propagating front could appear.

SL's method of analysis, in which the field variables are expanded in powers of time, has the advantage that it represents the complex interaction between the flow and the density structure as a series of stages. Timescales for frontogenesis can be correctly deduced from this analysis, but to obtain details of the final approach to the appearance of a front requires a numerical solution of the evolution equations.

I wish to thank Dr P. F. Linden for his useful and encouraging comments on a previous draft of this paper; Dr P.C. Matthews also made useful comments on possible applications of my theoretical ideas. I am grateful to Dr E. C. Carmack for permission to reproduce his diagram as figure 11 .

\section{REFERENCES}

Carmack, E. C. 1979 Combined influence of inflow and lake temperatures on Spring circulation in a riverine lake. J. Phys. Oceanogr. 9, 422-434.

Cormack, D. E., Leal, L. G. \& Imberger, J. 1974 Natural convection in a shallow cavity with differentially heated end walls. Part 1. Asymptotic theory. J. Fluid Mech. 65, 209-229.

Elliot, G. H. 1971 A mathematical study of the thermal bar. In Proc. 14th Conf. Great Lakes Res., pp. 545-554. Ann Arbor, Michigan: Intl Assoc. Great Lakes Res.

Foster, T. D. 1972 An analysis of the cabbeling instability in sea water. J. Phys. Oceanogr. 2, 294-301.

Hoskins, B. J. 1982 The mathematical theory of frontogenesis. Ann. Rev. Fluid Mech. 14, $131-151$.

Hubbard, D. W. \& Spain, J. D. 1973 The structure of the early Spring thermal bar in Lake Superior. In Proc. 16th Conf. Great Lakes Res., pp. 735-742. Ann Arbor, Michigan : Intl Assoc. Great Lakes Res.

Ivey, G. N. \& Hamblin, P. F. 1989 Convection near the temperature of maximum density for high Rayleigh number, low aspect ratio, rectangular cavities. Trans. ASME C: J. Heat Transfer 111, 100-105.

JACQMin, D. 1991 Frontogenesis driven by horizontally quadratic distributions of density. J. Fluid Mech. 228, 1-24.

KAY, A. 1990 Advection-diffusion in reversing and oscillating flows: 1. The effect of a single reversal. IMA J. Appl. Maths 45, 115-137.

LINDEN, P. F. \& Simpson, J. E. 1986 Gravity-driven flows in a turbulent fluid. J. Fluid Mech. 172, $481-497$ (referred to herein as LS).

Marmoush, Y. R., Smith, A. A. \& Hamblin, P. F. 1984 Pilot experiments on thermal bar in lock exchange flow. J. Energy Engng ASCE 110, 215-227.

Matthews, P. C. 1988 A model for the onset of penetrative convection. J. Fluid Mech. 188, 571-583.

Patterson, J. C. 1984 Unsteady natural convection in a cavity with internal heating and cooling. J. Fluid Mech. 140, 135-151.

Patterson, J. C. \& Imberger, J. 1980 Unsteady natural convection in a rectangular cavity. J. Fluid Mech. 100, 65-86.

Simpson, J. E. 1982 Gravity currents in the laboratory, atmosphere and ocean. Ann. Rev. Fluid Mech. 14, 213-234.

Simpson, J. E. \& Linden, P. F. 1989 Frontogenesis in a fluid with horizontal density gradients. J. Fluid Mech. 202, 1-16 (referred to herein as SL).

Sмгтн, G. D. 1985 Numerical Solution of Partial Differential Equations: Finite Difference Methods, 3rd Edn. Oxford University Press.

TAYLOR, G. I. 1953 Dispersion of soluble matter in solvent flowing slowly through a tube. Proc. $R$. Soc. Lond. A 219, 186-203. 